\title{
Article
}

Makoto Watanabe*

\section{Middlemen: A Directed Search Equilibrium Approach}

https://doi.org/10.1515/bejm-2019-0258

Received December 23, 2019; accepted May 9, 2020

\begin{abstract}
This paper studies an intermediated market operated by middlemen with high inventory holdings. I present a directed search model in which middlemen are less likely to experience a stockout because they have the advantage of inventory capacity, relative to other sellers. The model explains why the empirical relationship between middlemen's premium and their inventory capacity can be positive in some markets (e. g., rental video shops, used-car dealers) and negative in other markets (e. g., supermarkets, theater ticket offices). I also examine the implication of the configuration of middlemen's market in terms of the size and the number of middlemen for the equilibrium premium with and without free entry.
\end{abstract}

Keywords: directed search, intermediation, inventory holdings, retail markups

JEL Classification Number: D4, F1, G2, L1, L8, R1

\section{Introduction}

This paper presents a simple search model which allows us to study explicitly the dependence of middlemen's premium on their immediacy service backed by inventory capacity. It is not only that middlemen or dealers are viewed as the suppliers of immediacy to the market as in Stigler (1964), but also that the

\footnotetext{
I thank Melvyn G. Coles and participants in various seminars and conferences for comments and suggestions. This paper is a substantially revised version of the article circulated under the title "Middlemen: the bid-ask spread".
}

*Corresponding author: Makoto Watanabe, Department of Economics, School of Business and Economics, VU Amsterdam, De Boelelaan 1105, NL-1081 HV, Amsterdam, The Netherlands, E-mail: makoto.wtnb@gmail.com 
compensation to middlemen is captured by "the markup that is paid for predictable immediacy of exchange in organized markets; in other markets, it is the inventory markup of retailer or wholesaler."1

Using a directed search approach, Watanabe (2010) considers retail markets that are operated by middlemen and sellers who differ in the selling capacity. However, Watanabe (2010) focuses attention on turnover behavior of sellers to become middlemen, and so the behavior of retail premium has never been analyzed explicitly. ${ }^{2}$ In this economy, buyers have limited search time and their search activities are on an uncoordinated basis. Under these frictions, the middlemen's advantage in the selling capacity provides buyers with a lower likelihood of experiencing a stockout. Thus, the price difference between sellers and middlemen determines the retail premium charged for the more sure service rate, i. e., the immediacy service.

My approach offers a natural link between inventory capacity and immediacy. I show that the middlemen's inventory capacity has non-trivial effects on prices. On one hand, a larger inventory makes it less likely that excess demand occurs at individual middlemen, generating a downward pressure on the middlemen's price. On the other hand, it influences the search decision of buyers: a larger capacity of a middleman can attract more buyers. This effect creates a tighter market that allows to charge a higher price, since the middleman knows that buyers receive zero payoff in the event of a stockout. These conflicting effects cause a non-monotonic response of the price to capacities: it goes up (down) when the initial total supply is scarce (abundant). This is because the stockout probability is initially high (low) in the former (latter) situation, thereby buyers are prepared to pay a higher premium for a larger inventory when the the initial scarcity of resources is higher.

The empirical relationship between middlemen's premium and their inventory capacity can be positive in some markets (e. g., rental video shops, used-car

1 Page 36 in Demsetz (1968).

2 Another difference is that Watanabe $(2010,2018)$ present a special case of the current model assuming myopic agents, i. e., infinite discounting, and limited production technologies of sellers where middlemen can restock only from those sellers who remain their unit unsold in the retail market. Under these assumptions, Watanabe (2010) shows that turnover equilibria can be multiple - one is stable and has many middlemen, each with few units and a high price, and the other is unstable and has few middlemen, each with many units and a low price. The current paper generalizes these two assumptions. While it is true that things could get complicated easily, I offer a fairly simple way to pursue this generalization. On the other hand, with flexible production technologies by sellers in frictionless wholesale markets, the type of multiplicity discussed in Watanabe (2010) is hard to obtain. Hence, how middlemen stock inventory matters - this is not a model of homogeneous good producers of different size but of middlemen. 
dealers) ${ }^{3}$ and negative in other markets (e. g., supermarkets, theater tickets offices). ${ }^{4}$ Our result suggests that the former (latter) is likely to occur when the initial capacity of individual middlemen and total supply are relatively low (high).

The main insight can be generalized by considering the extensive margin of middlemen. With fixed supply in the middlemen's market, a larger individual capacity should accompany fewer middlemen. This setup allows me to show that the concentration of middlemen's market, i. e., having fewer middlemen, each with larger capacity, leads to a higher retail premium when the total supply is small, but can lead to a lower retail premium when the total supply is relatively large. With free entry of middlemen, when the inventory cost is low, there are many operating middlemen. This creates a situation of abundant total supply when the individual capacity level is high enough. In this situation, buyers would not appreciate much higher capacities and so the premium they are willing to pay for middlemen can decrease as the middlemen's market gets more concentrated. Conversely, when the capacity level is low, the total supply

3 Dana and Spier (2001) conduct a survey about four new releases at 20 rental video outlets within a local market and find that Blockbuster Video (the leading intermediary in the rental video industry, who had adopted the policy to increase its stocks of new-releases) charged $\$ 3.81$ and had $86 \%$ availability on average, whereas the corresponding numbers were $\$ 3.32$ and $60 \%$ for other national chains, and $\$ 2.62$ and $48 \%$ for the independent stores. A similar suggestive evidence can be found in used car markets. According to the "Used Car Market Report in 2007," produced by Manheim Consulting, about $65.7 \%$ of the 42.6 million U.S. used car sales in 2006 were from dealers. The average transacted price of a used vehicle was $\$ 10,875$ for franchised dealers, $\$ 8675$ for independent dealers, and $\$ 4450$ for private sellers. They note that the main reason for these differences was that large-scale dealers have greater control over their used vehicle inventory. 4 First of all, Aguirregabiria (2005) finds that intermediaries' inventory is a critical variable to explain their pricing patterns, especially when customers trade-off the price against the service rate. Further, Aguirregabiria (1999) identifies a negative effect of inventory ordering on the markups in a supermarket chain. In connection with the latter evidence, retailers' price increases following supermarket leveraged buy-outs (LBOs) are observed in Chevalier (1995) and Matsa (2011). All these empirical findings are consistent with the effect of capacities on retail prices illustrated in my model, given that high leverage may lead firms to be cash-constrained and thus may reduce their ability to maintain the available stock for future sales. A related evidence provided by Matsa (2009) shows that stockouts are negatively correlated with competition: supermarkets that face significantly high competition offer on average 5 percent lower stockout rates than otherwise similar stores. This means that, as is consistent with the case of relatively high capacities in my model, a higher competition in the presence of large-scaled retailers, like Wal-Mart, is associated with a lower stockout probability offered to their customers. My model captures the fact that when intermediaries face the risk of excessive amount of unsold inventories, they often have a large price reduction in last minute. Such an evidence can be found for instance in Leslie (2004) who finds in Broadway performances that 197 out of the 199 performances offered discounts of up to $50 \%$ on the day of the performance and that these discounts were applied to $14 \%$ of the attendants. 
can be scarce enough for the premium to be increasing. Hence, with free entry, the retail premium can be non monotone in the capacity when the inventory cost is low.

The rest of the paper is organized as follows. This introductory section closes with more detailed discussions on the related literature. Section 2 presents the basic setup and studies the steady state equilibrium allocations. Section 3 provides the characterization of the retail price differentials. Section 4 extends the analysis to allow for the free entry of middlemen. Section 5 concludes. All the proofs are in the Appendix.

\subsection{Related Literature}

This study contributes to the literature of middlemen, initiated by Rubinstein and Wolinsky (1987), that emphasizes the middlemen's advantage over the original suppliers in the rate at which they meet buyers (see also Awaya, Chen and Watanabe, 2018; Awaya, Iwasaki, and Watanabe 2019; Geromichalos and Jung 2018; Lagos and Zhang 2016; Masters 2007; Nosal, Wong, and Wright 2015; Watanabe 2010, 2018; and Wong and Wright 2014). In Rubinstein and Wolinsky (1987), it is assumed that: (i) the matching rates of agents are exogenous; ${ }^{5}$ (ii) the terms of trades are determined by Nash bargaining; ${ }^{6}$ (iii) middlemen can hold only one unit of a good as inventory. In contrast, my approach is based on a standard directed search equilibrium ${ }^{7}$ and allows me to study both the matching advantage of middlemen and its influence on their market power, because it incorporates:

5 Given some exogenous meeting process, two main reasons have been considered for the middlemen's advantage in the rate of successful trades: a middleman may be able to guarantee the quality of goods (Biglaiser 1993; Biglaiser and Li 2018; Li 1998), or to satisfy buyers' demand for a variety of goods (Shevichenko 2004).

6 In another approach used in Gehrig (1993), Spulber (1996), and Rust and Hall (2003) (see also the book by Spulber (1999)), price setting is emphasized as the middlemen's main role of marketmakings, but the meeting rate is exogenous. For further issues, see, for instance, Fingleton (1997a, 1997b), Caillaud and Jullien (2003), Smith (2004), Galeotti and Moraga-Gonzalez (2009), MoragaGonzalez and Wildenbeest (2012), Condorelli, Galeotti, and Skreta (2018) and Rhodes, Watanabe, and Zhou (2019).

7 In the directed search literature, Moen (1997), Mortensen and Wright (2002), and Sattinger (2003) consider fictitious market makers (not middlemen), who replace the Warlasian auctioneer, to interpret the efficiency property of competitive search equilibrium: Given the market makers create different submarkets and announce a pair of price and service probability in each submarket, buyers and sellers are willing to trade in a submarket where the Hosios condition holds endogenously. The latter property holds true in the equilibrium with middlemen constructed in my model. An efficiency/welfare analysis will be available upon request. 
(i) buyers' choice of where to search so that the matching rate between buyers and suppliers is determined endogenously; (ii) competition among suppliers so that individual suppliers can influence the search-purchase behaviors of buyers through prices; (iii) middlemen's inventory holdings of more than one unit so that the dependence of both the buyers' search decision and the extent of competition on their inventory can be made explicit.

In a recent paper, Gautier, $\mathrm{Hu}$ and Watanabe (2018) consider a simplified version of the model where the middlemen's inventory is modeled as a mass, not as an indivisible unit as in the current paper, assuming more flexible inventory technologies, so that the middleman faces a degenerate distribution of sales. This simplification gives tractability which allows them to study the choice of intermediation modes: an intermediary can operate as a middleman (a merchant or a dealer) to pursue buying and selling, and/or as a market-maker (a platform or a broker) to be engaged in fee business by offering a marketplace. A similar simplification is used in Holzner and Watanabe (2018) which studies a jobbrokering service offered by Public Employment Agencies (PEA) whose ability to facilitate the matching between the registered job seekers and vacancies is similar to the middlemen's high capability to deal with many agents simultaneously. ${ }^{8}$ However, with this simplification, frictions disappear in the middlemen's market and so the non monotonicity of intermediation premia can never occur.

This paper is also related to the recent literature on financial intermediaries pioneered by Duffie, Garleanu, and Pedersen (2005). They use a bargainingbased search model, together with time varying preference shocks, to formulate the trading frictions that are characteristic of over-the-counter markets. On the methodological side, there are situations in which the notion of directed search might provide a better description of pricing and trading mechanisms than the standard model with bargaining and random search. For instance, the Securities and Exchange Commission Rule 11Ac1-5, later redesignated as Rule 605 of the National Market System Regulation, requires market centers to report various execution quality statistics (so called Dash 5 reports) in publicly traded securities. The information made available by each market center includes time of execution and trading costs (such as effective bid-ask spreads) on a stock-bystock basis. Similarly, broker/dealers in over the counter secondary market have an obligation to report transactions in corporate bonds to the Trade Reporting and Compliance Engine. This type of information is consistent with the notion of

8 Holzner and Watanabe (2018) also find empirically that mitigating coordination frictions is the primary role of the PEA. See also Li et al. (2019) for another empirical attempt of testing implications of directed search models of intermediation. 
directed search. Further, analyzing execution quality on Nasdaq and the NYSE using the Dash-5 data, Boehmer (2005) finds that high execution costs are systematically associated with fast execution speed, and low costs are associated with slow execution speed. This relationship holds both across markets and across order sizes. This is the key trade-off that is common in directed search equilibria. In a recent paper, Lester, Rocheteau, and Weill (2014) offer a version of Duffie et al. (2005) with publicly observable prices but not from the perspective of middlemen's inventory holdings. Unlike my paper, they do not study the behavior of retail premia and its dependence on the immediacy service middlemen offer.

\section{Model}

Consider an economy inhabited by a continuum of homogeneous buyers, sellers and middlemen, indexed $b, s$ and $m$, respectively. The population of buyers is normalized to one, and the population of sellers and middlemen are denoted by $S$ and $M$, respectively. These population parameters are constant over time. All agents are risk-neutral and infinitely lived. Time is discrete and each period is divided into two subperiods. During the first subperiod, a retail market is open for a homogeneous, indivisible good to buyers. The good is storable. This retail market is operated by sellers and middlemen, and is subject to search frictions as described in detail below. Each period, each buyer has unit demand while each seller has a capacity $k_{s}=1$, and each middleman has a capacity $k_{m} \geq 1$ units of the good. The selling capacity of suppliers $k_{i}$ is exogenously given, for both $i=s, m$. The consumption value of the good is normalized to unity. If a buyer successfully purchases in the retail market at a price $p$, then he obtains the per-period utility of one. Otherwise, he receives zero utility that period. A seller or a middleman who sells $z$ units at a price $p$ obtains the revenue $z p$ during the first subperiod.

During the second subperiod, a wholesale market opens. This market is operated by sellers, and middlemen can restock their units for the future retail markets. ${ }^{9}$ There is no search friction in the wholesale market and the price is determined competitively. Sellers decide whether to produce for the current

\footnotetext{
9 The timing of events is irrelevant here - the entire analysis remains unchanged with an alternative setting in which the retail market occurs in the second subperiod or production occurs in the retail market rather than in the restocking market. Also, one could alternatively assume that buyers own an income measured in terms of numeraire (or a compound good) and use it for buying the good in question. Here, I have selected the simplest possible presentation.
} 
wholesale market and for the future retail market. Sellers can produce any units they wish for any purposes. The production cost is measured in terms of utility and is given by $c<1$ per unit. Agents discount future payoffs at a rate $\beta \in[0,1)$ across periods, but there is no discounting between the two sub-periods.

In each retail market, sellers and middlemen simultaneously post a price which they are willing to sell at. Observing the prices, all buyers simultaneously decide which seller or middleman to visit. Each buyer can visit one seller or one middleman. If more buyers visit a seller or middleman than its selling capacity, then the unit or units are allocated randomly. Assuming buyers cannot coordinate their actions over which seller or middleman to visit, a directed-search equilibrium is investigated where all buyers use an identical strategy for any configuration of the announced prices. I focus my attention on a stationary directed-search equilibrium where all sellers post the identical price $p_{s}$ and all middlemen post the identical price $p_{m}$ every period.

In any given period, each individual seller or middleman is characterized by an expected queue of buyers, denoted by $x$. The number of buyers visiting a given seller or middleman who has expected queue $x$ is a random variable, denoted by $n$, which has the Poisson distribution, Prob $\cdot(n=k)=\frac{e^{-x} x^{k}}{k !}$. In a directed-search equilibrium where $x_{i}$ is the expected queue of buyers at $i$, each buyer visits some seller (and some middleman) with probability $S x_{s}$ (and $M x_{m}$ ). They should satisfy the adding-up restriction,

$$
M x_{m}+S x_{s}=1
$$

requiring that the number of buyers visiting individual sellers and middlemen be summed up to the total population of buyers. The buyer's probability of being served by a supplier $i$, conditional on visiting it, depends on the queue $x_{i}$ and the selling capacity $k_{i}$. Denote this probability by $\eta\left(x_{i}, k_{i}\right)$. It is computed as follows: ${ }^{10}$

$$
\eta\left(x_{i}, k_{i}\right)=\frac{\Gamma\left(k_{i}, x_{i}\right)}{\Gamma\left(k_{i}\right)}+\frac{k_{i}}{x_{i}}\left(1-\frac{\Gamma\left(k_{i}+1, x_{i}\right)}{\Gamma\left(k_{i}+1\right)}\right)
$$

where $\Gamma(k, x)=\int_{x}^{\infty} t^{k-1} e^{-t} d t . \eta(\cdot)$ is strictly decreasing (increasing) in $x_{i}$ (in $k_{i}$ ). For $i=s, \eta\left(x_{s}, 1\right)=1-e^{-x_{s}} / x_{s}$ is a standard urn-ball matching function.

In steady state, each seller holds $k_{s}=1$ unit and each middleman holds $k_{m} \geq 1$ units at the start of every period. As the wholesale market is competitive, middlemen

10 See Watanabe (2018) for the derivation of this probability and the expected number of sale by a supplier $i$ given by $x_{i} \eta\left(x_{m}, k_{m}\right)$ (not by the use of a "law of large number"). See also Watanabe (2010) which shows that the market equilibrium presented in this section is identical to the limiting solution in a finite setup counterpart as the population gets large. 
can restock at the sellers' reservation price $c{ }^{11}$ Simultaneously, sellers produce another unit for the next retail market, if the production cost is not too high, and if they have successfully sold in the retail market - holding more than one unit is never optimal since sellers only have the selling capacity of $k_{s}=1$ in the retail market.

I now characterize the equilibrium retail prices. In any equilibrium where $V^{b}$ is the value of a buyer and where middlemen restock at the sellers' reservation price $c$, the optimal retail price of a supplier $i$ who has a capacity $k_{i}$ and an expected queue of buyers $x$, denoted by $p_{i}\left(V^{b}\right)$, satisfies

$$
p_{i}\left(V^{b}\right)=\operatorname{argmax}_{p}\left[x \eta\left(x, k_{i}\right)(p-c)\right],
$$

subject to

$$
V^{b}=\eta\left(x, k_{i}\right)(1-p)+\beta V^{b} .
$$

For $i=s$, a seller sells its good at price $p$ with probability $x \eta(x, 1)$, and produces a new unit with cost $c$ for the next period. If unsuccessful in the retail market, then the seller's payoff is zero: if it sells the current unit to a middleman in the second subperiod, then it receives $c$ from the middleman and produces a new unit for the next period with $\operatorname{cost} c$; otherwise, the seller receives nothing and carries its unit into the next period. For $i=m$, a middleman's expected number of sales is $x \eta\left(x, k_{m}\right)$, and it restocks at the competitive price $c$. (2) says that the supplier $i$ with a price $p$ and an expected queue $x$ must deliver to buyers at least their market payoff $V^{b}$, and clearly it does not deliver more. While $V^{b}$ is an equilibrium object (i. e., determined below by equilibrium), it is taken as given by individuals. Notice that (2) generates tradeoffs between $p$ and $x$ : buyers can get the same $V^{b}$ from higher $p$ if $x$ is lower. A buyer choosing $p$ is served with probability $\eta\left(x, k_{i}\right)$ in which case he obtains per-period utility $1-p$. If not served by the seller or middleman, the buyer's payoff is zero that period. Irrespective of whether or not to be served, he enters the next period and his continuation value is $\beta V^{b}$. The situation is the same for all the other buyers.

Substituting out $p$ using (2), $p=1-\frac{1-\beta}{\eta(\cdot)} V^{b}$, the objective function of a supplier $i$, denoted by $\Pi_{i}(x)$, can be written as

$$
\Pi_{i}(x)=x \eta\left(x, k_{i}\right)(1-c)-x(1-\beta) V^{b} .
$$

11 Below, it is assumed that the capacity constraint of suppliers is binding for a middleman, so that he accumulates inventories up to the limit $k_{m}$. In financial markets, it can be justified by the reserve/capacity requirement. In other markets, one can assume a significantly high costs of maintaining an unfilled capacity or displaying unfilled shelves due to reputation concerns. To endogenize $k_{m}$ is technically more involved with the current formulation but can be done with the simplification as in Gautier, $\mathrm{Hu}$, and Watanabe (2018). 
Note that pricing and restocking are separate decisions, and one can find an optimal price by maximizing the objective function $\Pi_{i}(x)$ with respect to $x$. The first-order condition is

$$
\frac{\partial \Pi_{i}(x)}{\partial x}=\left(\eta\left(x, k_{i}\right)+x \frac{\partial \eta\left(x, k_{i}\right)}{\partial x}\right)(1-c)-(1-\beta) V^{b}=0
$$

for $i=s, m .^{12}$ Rearranging the first order condition above using (2) and

$$
\frac{\partial \eta\left(x, k_{i}\right)}{\partial x}=-\frac{k_{i}}{x^{2}}\left(1-\frac{\Gamma\left(k_{i}+1, x\right)}{\Gamma\left(k_{i}+1\right)}\right),
$$

one can obtain the optimal price of the seller (if $i=s$ ) or the middleman (if $i=m$ ),

$$
p_{i}\left(V^{b}\right)=c+\varphi^{i}\left(x, k_{i}\right)(1-c)
$$

where

$$
\varphi^{i}\left(x, k_{i}\right) \equiv-\frac{\partial \eta\left(x, k_{i}\right) / \partial x}{\eta\left(x, k_{i}\right) / x}
$$

is the elasticity of the matching rate of buyers, which represents the supplier $i$ 's share of the net trading surplus $1-c$.

The analysis above has established the equilibrium prices $p_{i}\left(V^{b}\right)$ given $V^{b}$. Equilibrium implies buyers are indifferent between any of the individual suppliers $i=s$, $m$, leading to

$$
\begin{aligned}
(1-\beta) V^{b} & =\eta\left(x_{s}, 1\right)\left(1-p_{s}\right) \\
& =\eta\left(x_{m}, k_{m}\right)\left(1-p_{m}\right),
\end{aligned}
$$

where $x_{i}$ is the equilibrium queue of buyers at $p_{i}=p_{i}\left(V^{b}\right), i=s, m$. Buyers successfully purchase the good from the seller or middleman with probability $\eta\left(x_{i}, k_{i}\right)$ each period. The value of sellers and middlemen are given by

$$
\begin{gathered}
(1-\beta) V^{s}=x_{s} \eta\left(x_{s}, 1\right)\left(p_{s}-c\right) \\
(1-\beta) V^{m}=x_{m} \eta\left(x_{m}, k_{m}\right)\left(p_{m}-c\right),
\end{gathered}
$$

12 The second-order condition is satisfied as it holds that for both $i=s, m$.

$$
\frac{\partial^{2} \Pi_{i}(x)}{\partial x^{2}}=-\frac{x^{k_{i}-1} e^{-\chi}}{\Gamma\left(k_{i}\right)}(1-c)<0 .
$$


respectively. Sellers produce with cost $c$ whenever needed, and middlemen restock at the competitive price $c$ each period. To solve for the equilibrium, it is important to observe that the indifference conditions (4) and (5) can be reduced to the following simple form: applying (3) for $i=s$ to (4) with a rearrangement,

$$
\frac{(1-\beta) V^{b}}{1-c}=\eta\left(x_{s}, 1\right)\left(1-\varphi^{s}\left(x_{s}, 1\right)\right)=e^{-x_{s}}
$$

similarly, applying (3) for $i=m$ to (5) with a rearrangement,

$$
\frac{(1-\beta) V^{b}}{1-c}=\eta\left(x_{m}, k_{m}\right)\left(1-\varphi^{m}\left(x_{m}, k_{m}\right)\right)=\frac{\Gamma\left(k_{m}, x_{m}\right)}{\Gamma\left(k_{m}\right)} ;
$$

these two equations together imply

$$
\frac{\Gamma\left(k_{m}, x_{m}\right)}{\Gamma\left(k_{m}\right)}=e^{-x_{s}} .
$$

The adding-up restriction (1) and the indifference condition (8) identify an equilibrium allocation $x_{s}, x_{m}>0$.

Theorem 1: (Stationary directed-search equilibrium).

Given $c \in[0, \bar{c}]$, some $\bar{c} \in(0, \beta)$, a stationary directed search equilibrium exists for all $\beta \in[0,1), S \in(0, \infty), M \in(0, \infty) k_{m} \geq 1$, with a unique market outcome satisfying $V^{b} \in(0,1-c / 1-\beta), x_{s} \in(0,1 /(S+M)], x_{m} \in[1 /(S+M), 1 / M), p_{i} \in(c, 1)$, and $V^{i} \in\left(0, k_{i}(1-c) / 1-\beta\right), i=s, m$.

This theorem is a straightforward generalization of Watanabe $(2010,2018)$ which present a special case of the current model assuming myopic agents, i. e., infinite discounting. At the start of each period, each seller holds one unit and each middleman holds $k_{m} \geq 1$ units in the retail market. Sellers produce for the retail market given that production costs are not that high $c \leq \bar{c}$, and middlemen restock in the competitive wholesale market each period. The equilibrium allocation of buyers $x_{s}, x_{m}>0$ is determined irrespective of the discount factor $\beta$ and production cost $c$ each period by (1) and (8). When $k_{m}=1$, there is no difference between sellers and middlemen in the retail market and so all sellers and middlemen receive the identical number of buyers $x_{s}=x_{m}$ and post the identical price $p_{s}=p_{m}$. Using the indifference condition (8), combined with the adding up restriction, one can show that a supplier with a larger capacity should accommodate more buyers. Hence, an increase in the middlemen's capacity $k_{m}$ induces more buyers to visit middlemen and fewer buyers to visit sellers, resulting in an increase in $x_{m}$ and a decrease in $x_{s}$. An increase in the proportion of sellers $S$ or middlemen $M$ leads to a fewer number of buyers per each supplier, which decreases $x_{s}, x_{m}$. 


\section{Retail Market Prices}

In this section, I study the behaviors of the retail prices. I begin by showing that the usual market-tightness effect leads to a lower price.

Proposition 1: (Market tightness effect) An increase in the population of sellers $S$ or middlemen $M$ (relative to that of buyers) leads to a lower retail market price $p_{i}, i=s, m$.

The market-tightness effect implies that a larger supply makes the retail market less tight and more competitive, leading to a lower retail price. This is standard in directed search models, but it is important that show that it also applies to our economy with middlemen especially to understand the nonmonotonicity result described below.

I now investigate the comparative statistics results of middlemen's capacity $k_{m}$ on the retail prices. In the current framework, the retail price differential, i. e., the price difference between sellers and middlemen in the retail market, is given by

$$
p_{m}-p_{s}=\left[\varphi^{m}\left(x_{m}, k_{m}\right)-\varphi^{s}\left(x_{s}, 1\right)\right](1-c),
$$

where $\varphi^{i}\left(x_{i}, k_{i}\right)$ represents the supplier $i$ 's share of the net trading surplus $1-c$ (see (3)).

Proposition 2: (Retail market premium). For all $S, M \in(0, \infty)$, the retail market premium of middlemen is zero when $k_{m}=1$ and is strictly positive when $1<k_{m}<\infty$.

When $k_{m}=1$, there is no difference between sellers and middlemen in the retail market, and so the premium is zero. When $k_{m}>1$, the price of middlemen is higher than that of sellers. The positive premium reflects the immediacy, or a relatively high rate of being served $\eta\left(x_{m}, k_{m}\right)>\eta\left(x_{s}, 1\right)$, that middlemen provide with its selling capacity $k_{m}>1$. Below, I show that the behavior of $p_{m}$ shapes critically that of the retail market premium.

The equilibrium price of middlemen is given by

$$
p_{m}=c+\varphi^{m}\left(x_{m}, k_{m}\right)(1-c)
$$

where

$$
\varphi^{m}\left(x_{m}, k_{m}\right)=\frac{1-\frac{\Gamma\left(k_{m}+1, x\right)}{\Gamma\left(k_{m}+1\right)}}{x_{m} \eta\left(x_{m}, k_{m}\right) / k_{m}}
$$

is the elasticity of matching function as mentioned above. Here, the denominator $x_{m} \eta(\cdot) / k_{m}$ represents the average probability of selling any given single unit, and the numerator,

$$
\operatorname{Prob} \cdot\left(n>k_{m}\right)=\sum_{n=k_{m}+1}^{\infty} \frac{e^{-x_{m}} x_{m}^{n}}{n !}=1-\frac{\Gamma\left(k_{m}+1, x_{m}\right)}{\Gamma\left(k_{m}+1\right)},
$$


represents the stock-out probability of an individual middleman - the probability that the number of buyers visiting the middleman $n$ is strictly greater than its capacity $k_{m} \cdot{ }^{13}$ The stockout probability is decreasing in the capacity $k_{m}$ and is increasing in the queue of buyers $x_{m}$.

There are two important effects of an increase in the capacity of middlemen $k_{m}$ on their price $p_{m} \cdot{ }^{14}$ On the one hand, a larger capacity of a middleman implies a smaller likelihood of excess demand and a smaller stockout probability of the middleman, given $x_{m}$. On the other hand, an increase in $k_{m}$ implies an increase in the number of buyers to visit middlemen, rather than sellers. The latter effect makes the middlemen's market tighter and increases the stockout probability of individual middlemen. Suppose that the latter effect is large enough to make the price of middlemen increase with their capacity. Then, it means that middlemen can extract a larger share of trading surplus from buyers, since they know that buyers receive zero payoff in the event of stockouts. Conversely, suppose that the increase in $x_{m}$ is relatively small, so that a middleman has a smaller likelihood of successfully selling out its entire units and its stockout becomes less likely. Then, buyers can receive a larger surplus share per unit, since buyers know that the middleman receives zero payoff from unsold units. Hereafter, I assume $S=1$ to simplify the analysis. Denote by

$$
X \equiv \frac{1}{M k_{m}+1}<1
$$

the per-period ratio of the total demand to the total supply in the retail market.

Proposition 3: (Retail market prices/differential).

Assume fixed values of $S=1$ and $M \in(0, \infty)$.

1. The retail price of middlemen $p_{m}$ is increasing in sufficiently low $k_{m}$, if and only if $X>X^{\star} \in(0,1)$, and is decreasing in sufficiently large $k_{m}$ for any given $X \in(0,1)$.

2. The retail price of sellers $p_{s}$ is decreasing in all $k_{m}$, for any given $X \in(0,1)$.

3. The retail market differential $p_{m}-p_{s} \geq 0$ is increasing in sufficiently low $k_{m}$ and is decreasing in sufficiently large $k_{m}$ for any given $X \in(0,1)$.

Figure 1 plots the behaviors of the price $p_{m}$ and Figure 2 the behaviors of the price differential $p_{m}-p_{s}$ in response to changes in the middlemen's capacity $k_{m}$, for given values of $M$ (and hence $\left.X\right) .{ }^{15}$ The non-monotonicity occurs because more buyers visit middlemen as their capacity increases - without the increase in $x^{m}$ that leads to a tighter middlemen's market, the price

13 The second equation follows from the series definition of cumulative gamma function, $\sum_{n=0}^{k} e^{-x} x^{n} / n !=\Gamma(k+1, x) / \Gamma(k+1)$.

14 Obviously, these two effects increase buyers' welfare.

15 In all the figures, I set $\beta=0.95$ and $c=0.2$. 
increase would be impossible (Proposition 1). For relatively small $k_{m}$, the stockout probability is relatively high given values of $x_{m}$. Since the increase in the number of buyers visiting middlemen $x_{m}$ is sufficiently large when the total demand $X$ is high, an increase in $k_{m}$ can make a tighter middlemen's market and result in a higher price $p_{m}$, if $k_{m}(X)$ is initially low (high). For relatively large $k_{m}$, the total demand ratio is relatively low and the stockout probability is already low. In this situation, a larger $k_{m}$ leads to an increase in the total supply that makes it less likely that an excess demand occurs at individual middlemen, resulting in a lower price. Unlike $p_{m}$, the sellers's price $p_{s}$ is monotone decreasing in $k_{m}$, because the sellers' market gets less tight as $k_{m}$ increases. This implies, the non-monotonicity of the retail market differential, $p_{m}-p_{s}$, is driven by that of the middlemen's price: there is a larger (smaller) premium for a larger selling capacity of middlemen when the total demand ratio $X$ is initially high (low).

Notice that in the above analysis, changes in $k_{m}$ affect the total supply. To abstract it from the effects of changes in the total demand-supply ratio, I examine the same comparative statistics exercise but, this time, fixing the middlemen's total supply denoted by $G=M k_{m}$.

Proposition 4: (Fixed supply in middlemen's market).

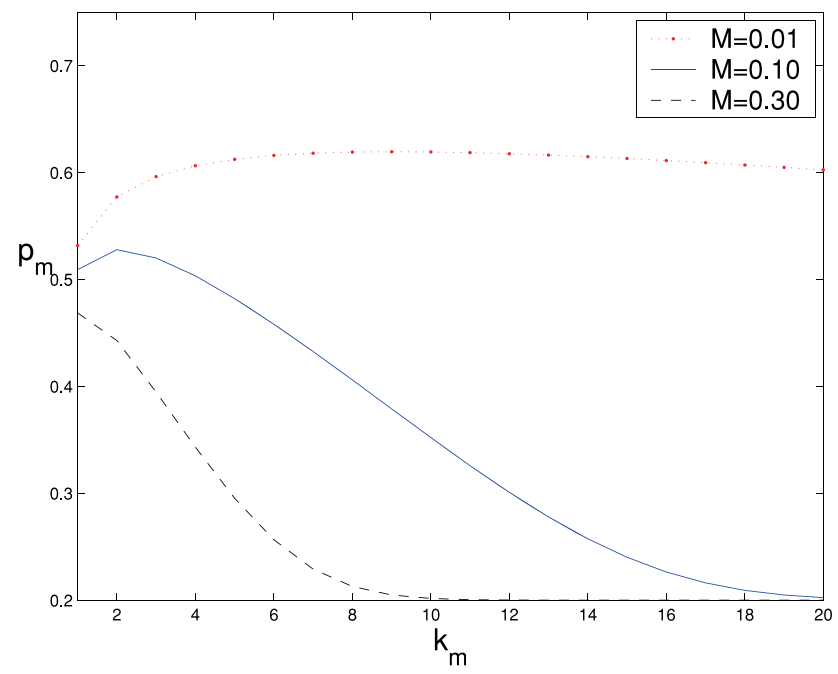

Figure 1: Retail price of middlemen. 


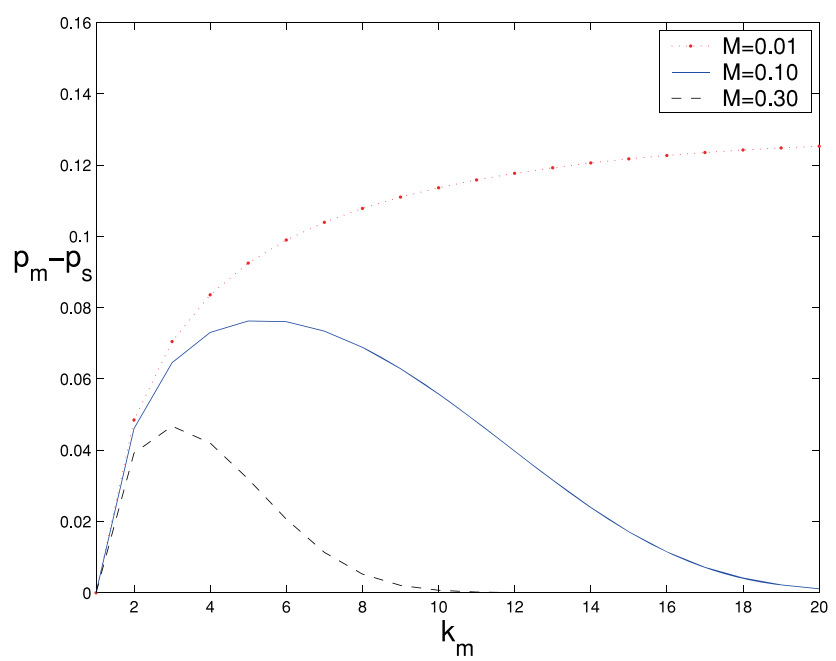

Figure 2: Retail price differentials.

Assume fixed values of $S=1$ and $G=M k_{m} \in(0, \infty)$.

1. The retail price of middlemen $p_{m}$ is increasing in sufficiently low $k_{m}$, if $X=1 / G+1>X^{\star} \in(0,1)$, and the retail market differential $p_{m}-p_{s} \geq 0$ is increasing in sufficiently low $k_{m}$ for any given $X \in(0,1)$.

2. The retail price of middlemen and the retail price differential are both decreasing in sufficiently large $k_{m}$ when $X<X^{* *} \in(0,1)$, and may or may not be decreasing in sufficiently high $k_{m}$, satisfying $p_{m}>c$ and $p_{m}-p_{s}>0$, when $X>X^{* *} \in(0,1)$.

Fixing total supply $G=M k_{m}$ generates two margins: one is the intensive margin (as already seen above) and the other is the extensive margin, where $M$ decreases with $k_{m}$. As the extensive margin implies a price increase, the item 1 in Proposition 4 shows that the condition of price increase with low $k_{m}$ is less stringent than before. The item 2 shows that with fixed total supply in the middlemen's market, the price of middlemen $p_{m}$ can stay above $c$ and the price differential can be positive $p_{m}-p_{s}>0$ even for large $k_{m}$, especially when the total demand $X$ is relatively high (which never happen with flexible supply). However, the essential insight remains valid: the concentration of middlemen's market, i. e., having fewer middleman, each with larger capacity, leads to a higher retail premium when $X$ is large, but can lead to a lower retail premium when $X$ is small. Figure 3 (a) plots the behavior of $p_{m}$ and Figure 3 (b) plots the behavior of price differential $p_{m}-p_{s}$, with different values of $X$. 
(a) Retail Price of Middlemen

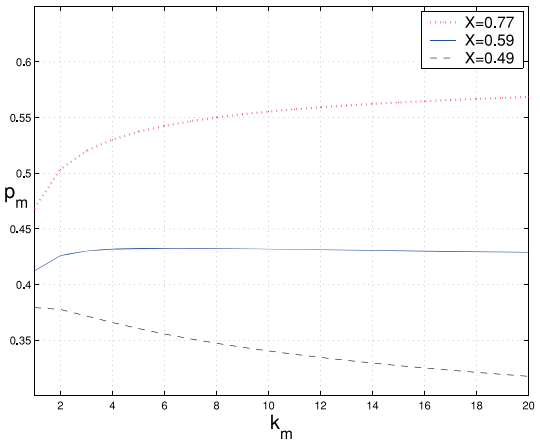

(b) Retail Price Differential

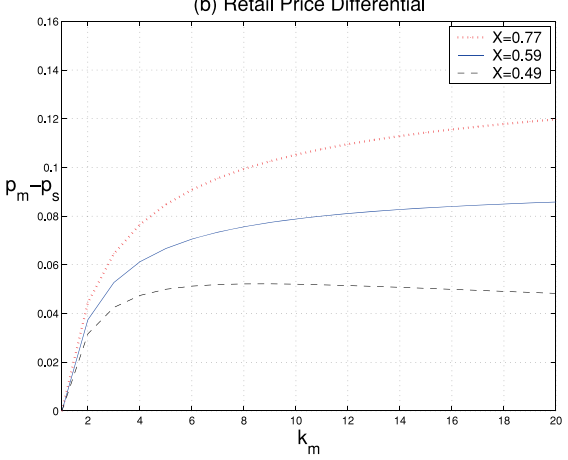

Figure 3: Concentration of middlemen's market.

\section{Free Entry Equilibrium}

In this section, I allow for the number of middlemen to be determined endogenously by free entry. So far, I have implicity assumed that middlemen hold the initial endowment $k_{m}$ at the start of their lifetime period. Suppose now that the initial endowment can be obtained by paying $c k_{m}>0$ from the sellers' market, whereas before $c>0$ represents the wholesale price. Middlemen possess the inventory management technologies that enable them to operate with a relatively large selling capacity in the retail market. Denote by $c_{k}$ the latter cost of inventory management per unit. This is a flow cost payed period by period. An agent chooses to be a middlemen if the value of being a middlemen is non-negative, $-\left(c+c_{k} / 1-\beta\right) k_{m}+V^{m} \geq 0$, given values of $k_{m} \geq 1$ and $M>0$. In a free entry equilibrium, entry and exit occur until the middlemen operating in the markets earn zero expected net profits, just to cover the cost. The equilibrium number of middlemen $M>0$ is determined by the free entry condition, $V^{m}=\left(c+c_{k} / 1-\beta\right) k_{m}$, or

$$
\left(1-\frac{\Gamma\left(k_{m}+1, x_{m}\right)}{\Gamma\left(k_{m}+1\right)}\right) \frac{1-c}{1-\beta}=c+\frac{c_{k}}{1-\beta},
$$

where the L.H.S. represents the per-unit profit of middlemen (apply the price in (3), $i=m$, to the value $V^{m}$ in (7)) and the R.H.S. the lifetime per-unit cost. As before, we consider low production costs, $c \in(0, \bar{c}]$, where $\bar{c}=\bar{c}(\bar{M}) \in(0, \beta)$ at some $\bar{M}<\infty$ (see the proof of Theorem 1), guaranteeing that seller produce for future sale under free entry of middlemen. Define the total per-unit cost per period as $C \equiv c(1-\beta)+c_{k} / 1-c$ and its upper bound as $\bar{C} \equiv \lim _{M \rightarrow 0} 1-\Gamma\left(k_{m}+1, x_{m}\right) /$ $\Gamma\left(k_{m}+1\right) \in(0,1)$. 
Theorem 2: (Free entry equilibrium). Given values of $C \in(0, \bar{C})$ with sufficiently low production costs, $c \in[0, \bar{c}]<\beta$, a free entry equilibrium exists, with a unique market outcome. In particular, the equilibrium number of middlemen $M \in(0, \infty)$ is: monotone decreasing in $C$; increasing in low $k_{m}$ if and only if $C>C^{\star} \in(0, \bar{C})$; decreasing in large $k_{m}$ for any $C \in(0, \bar{C})$.

The per-unit profit (i. e., L.H.S. of (9)) is decreasing in the number of middlemen, thereby a larger cost leads to fewer middlemen given values of $k_{m}$. Figure 4 plots the number of middlemen $M$ and $k_{m}$, for different values of $C$. The equilibrium number of middlemen can be non monotone in $k_{m}$ when $C$ is relatively high. Notice that the per-unit profit is proportional to the stockout probability, $1-\Gamma\left(k_{m}+1, x_{m}\right) / \Gamma\left(k_{m}+1\right)$, thereby a similar logic to the one stated before applies to generate the non-monotonicity of $M$ : when the total per-unit cost $C$ is high and the initial units $k_{m}$ are low, the number of middlemen is initially small and the total demand-supply ratio $X$ is initially high. In this situation, a larger $k_{m}$ can generate a tighter middlemen's market and increase the profitability of operating as a middleman. Hence, the number of middlemen increases with low $k_{m}$ when $C$ is high. The opposite happens when $C$ is low or $k_{m}$ is high, since in such a case, a larger $k_{m}$ makes it more likely that middlemen remain their units unsold, which reduces their profits and the number of operating middlemen.

In the free entry equilibrium with linear inventory cost, the stockout probability is kept constant by the free entry condition (9). This property leads to the following results.

Proposition 5: (Retail price/premium with free entry).

Consider the free entry equilibrium described in Theorem 2 given the linear inventory technology with parameter $C \in(0, \bar{C})$ and $S=1$.

1. The retail price of middlemen $p_{m}$ is decreasing in both small and large values of $k_{m}$, for any given $C \in(0, \bar{C})$.

2. The retail price differential $p_{m}-p_{s} \geq 0$ is increasing in sufficiently low $k_{m}$, for any given $C \in(0, \bar{C})$, and may or may not be decreasing in sufficiently large $k_{m}$, satisfying $p_{m}-p_{s} \rightarrow r_{O}(C)>0$, where $r_{O}(\cdot) \rightarrow O$ as $C \rightarrow O$.

As free entry implies a constant stockout probability with linear inventory cost, the item 1 in Proposition 5 shows that the price increase, which occurs without free entry for low $k_{m}$, disappears in the free entry equilibrium. Figure 5 (a) plots the behavior of $p_{m}$, where a larger capacity creates a competitive pressure and lowers the price. The item 2 in the proposition shows that $p_{m}-p_{s}$ approaches to zero when $k_{m}$ is sufficiently high and $C$ is sufficiently low. This implies a non monotonicity of the retail premium with respect to $k_{m}$ when $C$ is sufficiently low. Figure 5 (b) depicts the behavior of 


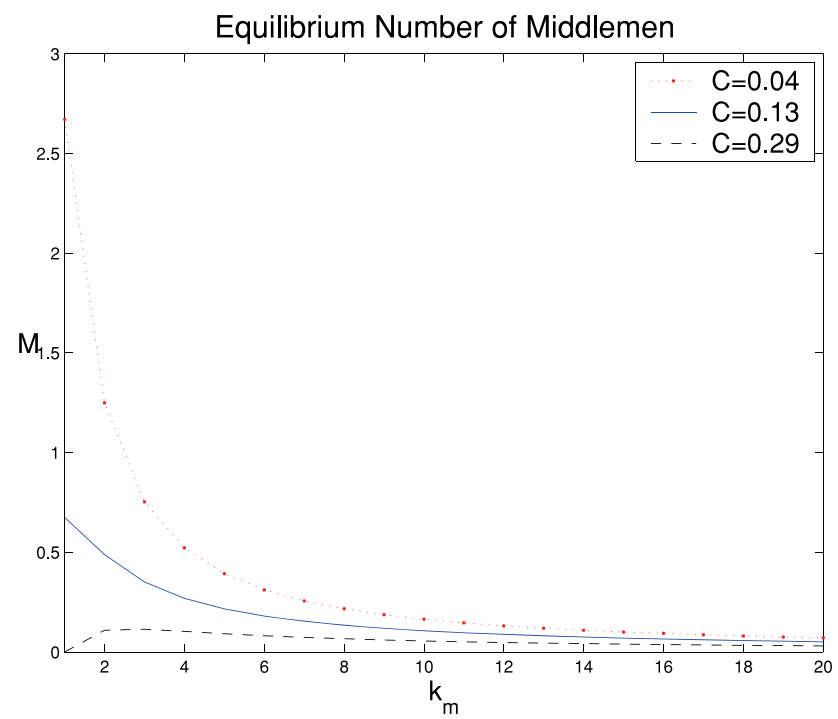

Figure 4: Free entry equilibrium.

price differential $p_{m}-p_{s}$. The emergence of the non-monotonicity can be understood by the same logic as before. When $k_{m}$ is high and $C$ is low, there are many operating middlemen each with large capacity and so the aggregate demand ratio $X=1 / M k_{m}+1$ is relatively low. In this situation, buyers would not appreciate much the capacity increase of middlemen so that the premium they are willing to pay for middlemen can decrease as the middlemen's market gets more concentrated. When $k_{m}$ is low or $C$ is high, the aggregate demand ratio is relatively high (i. e., the resource is relatively scarce in total). In this situation, the premium increases with capacities.

(a) Retail Price of Middlemen

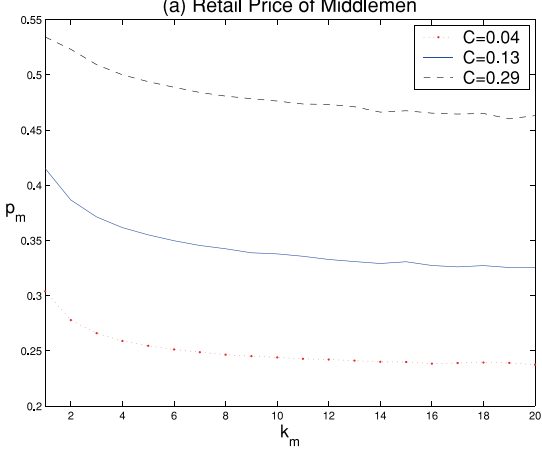

(b) Retail Price Differential

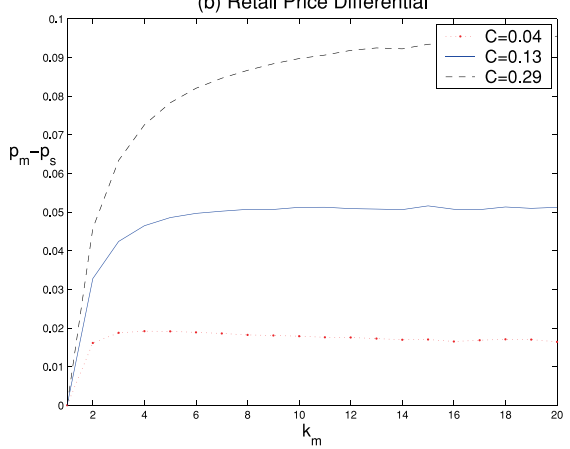

Figure 5: Retail price/premium with free entry. 


\section{Conclusion}

This paper proposed a simple theory of middlemen using a standard directed search approach. It offers wide applicability and economic insights into many empirically relevant forms of middlemen. Middlemen's inventories can provide buyers with immediacy service under market frictions, thereby the retail price of middlemen includes a premium to buyers. The model generates two important effects of middlemen's inventories that serve as the critical determinant of the retail premium. On the one hand, middlemen can attract more buyers with a larger selling capacity, which allows them to charge a higher price. On the other hand, it puts downward pressure on their retail price. These conflicting effects cause nonmonotonic responses of the retail price/premium to changes in their inventories. In particular, the premium goes up when the initial total supply is scarce, because buyers appreciate much the capacity increase and are prepared to pay a higher premium for a larger inventory. This result may help us understand why popular items are often sold at a larger premium by larger scaled intermediaries. The main insight survives with free entry of middlemen. With fixed supply in the middlemen's market, the concentration of middlemen's market, i. e., having fewer middlemen, each with larger capacity, leads to a higher retail premium when the total supply is small, but can lead to a lower retail premium when the total supply is relatively large.

For future research, it would be interesting to extend the model to allow for the middlemen's choice of payment method. In history, merchants and bankers, or enforcers, often share the same root. One implication of the retail premium analyzed here is that if buyers have to use cash for their retail transactions, they have to hold more cash to visit the middlemen's market than to visit the sellers' market. Hence, to the extent that cash holdings are costly, due to inflation or the cost of fraud, or because cash is susceptible to theft, middlemen have an incentive to introduce credit as a means of payment. In such an extended model, it would also be interesting to study the benefit and cost of making retail prices publicly observable, because it would determine the cash holdings of buyers in each market.

\section{Appendix}

\section{Proof of Theorem 1}

The proof takes three steps. Step 1 establishes a unique solution $x_{s}, x_{m}>0$ for all $k_{m} \geq 1, S \in(0, \infty)$ and $M \in(0, \infty)$, using (1), (3), (4) and (5). With a slight abuse of 
notation, let $x_{i}\left(k_{m}, S, M\right)$ denote this solution for $i=s, m$. Given this solution, Step 2 then identifies a unique solution $V^{j} \in(0,1-c / 1-\beta)$ to (4), (5) and (6) for $j=b, s$. The rest of the equilibrium values are identified immediately: given $x_{i}$, (3) determines a unique $p_{i} \in(c, 1)$ for $i=s, m$; given $x_{m}$ and $p_{m}$, (7) determines a unique $V^{m} \in\left(0, k_{m}(1-c) / 1-\beta\right)$. Hence, given the initial units $k_{i}, i=s, m$, for all $\beta \in[0,1)$, $k_{m} \geq 1, S \in(0, \infty), M \in(0, \infty), c \in[0, \bar{c}]$, this solution then satisfies (1), (3), (4), (5), (6), and (7). Finally, Step 3 shows that in any period, sellers produce a unit for the retail market given $c \leq \bar{c}$, some $\bar{c} \in(0, \beta)$. As middlemen restock their units in the Walrasian market each period, this implies all sellers hold $k_{s}=1$ unit and all middlemen hold $k_{m} \geq 1$ units at the start of each period, and so the established solution is indeed a steady state equilibrium.

\section{Step 1}

For any $k_{m} \geq 1, S \in(0, \infty)$ and $M \in(0, \infty)$, a solution $x_{i}=x_{i}\left(k_{m}, S, M\right)$ to (1), (3), (4) and (5) exists and is unique for $i=s, m$ that is: continuous in $S, M, k_{m} \in \mathbf{R}_{+}$; strictly decreasing in $S, M$; strictly increasing (or decreasing) in $k_{m}$ if $i=m$ (or if $i=s$ ) satisfying $x_{s}(1, \cdot)=x_{m}(1, \cdot)=1 /(S+M), x_{s}\left(k_{m}, \cdot\right) \rightarrow 0$ and $x_{m}\left(k_{m}, \cdot\right) \rightarrow 1 / M$ as $k_{m} \rightarrow \infty$.

Proof of Step 1. In the main text, it has been shown that (3), (4) and (5) imply (8). Substituting out $x_{m}$ in (8) by using (1),

$$
\frac{\Gamma\left(k_{m}, \frac{1-S x_{s}}{M}\right)}{\Gamma\left(k_{m}\right)}=e^{-x_{s}}
$$

where $\Gamma(k)=\int_{0}^{\infty} t^{k-1} e^{-t} d t$ and $\Gamma(k, x)=\int_{x}^{\infty} t^{k-1} e^{-t} d t$. The L.H.S. of this equation, denoted by $\Phi\left(x_{s}, k_{m}, S, M\right)$, is continuous and strictly increasing in $x_{s}$ and $k_{m} \in \mathbf{R}_{+}$, satisfying:

$$
\Phi\left(x_{s}, \cdot\right) \rightarrow \frac{\Gamma\left(k_{m}, \frac{1}{M}\right)}{\Gamma\left(k_{m}\right)}<1 \text { as } x_{s} \rightarrow 0 ; \Phi\left(\frac{1}{S+M}, \cdot\right)=\frac{\Gamma\left(k_{m}, \frac{1}{S+M}\right)}{\Gamma\left(k_{m}\right)} \geq e^{-\frac{1}{S+M}}
$$

with equality only when $k_{m}=1$;

$$
\Phi\left(x_{s}, 1, \cdot\right)=e^{-\frac{1-S_{S}}{M}} ; \Phi\left(x_{s}, k_{m}, \cdot\right) \rightarrow 1 \text { as } k_{m} \rightarrow \infty \cdot
$$

Similarly, $\Phi(\cdot)$ is continuous and strictly increasing in $S, M$ for any $x_{S} \in(0,1 /(S+M))$ and $k_{m} \geq 1$. It follows therefore that a unique solution $x_{S}=x_{S}\left(k_{m}, S, M\right) \in(0,1 /(S+M)]$ exists that is: continuous and strictly decreasing in $k_{m} \in[1, \infty) \subset \mathbf{R}_{+}$satisfying $x_{S}(1, \cdot)=1 /(S+M)$ and $x_{s}\left(k_{m}, \cdot\right) \rightarrow 0$ as $k_{m} \rightarrow \infty$; continuous and strictly decreasing in $S, M$.

Applying this solution to (1), one can obtain a unique solution $x_{m}=x_{m}\left(k_{m}, S, M\right) \in[1 /(S+M), 1 / M)$ that is: continuous and strictly decreasing in $S$ and $M$; continuous and strictly increasing in $k_{m} \in[1, \infty) \subset \mathbf{R}_{+}$satisfying 
$x_{m}(1, \cdot)=1 /(S+M)$ and $x_{m}\left(k_{m}, \cdot\right) \rightarrow 1 / M$ as $k_{m} \rightarrow \infty$. This completes the proof of Step 1.

\section{Step 2}

Given $x_{s} \in(0,1 /(S+M)]$ established in Step 1, there exists a unique solution $V^{j} \in(0,1), j=b, s$, to (3), (4), and (6).

Proof of Step 2. (3), (4), and (6) imply $V^{b}$ satisfies

$$
V^{b}=\frac{e^{-\chi_{s}}(1-c)}{1-\beta}
$$

The R.H.S of this equation, denoted by $Y_{b}\left(x_{s}\right)$, is strictly decreasing in $x^{s} \in(0, \infty)$ and satisfies: $Y_{b}(\cdot) \rightarrow 1-c / 1-\beta$ as $\chi_{s} \rightarrow 0 ; \Upsilon(\cdot) \rightarrow 0$ as $x_{s} \rightarrow \infty$. As equilibrium implies $x_{s} \in(0,1 /(S+M)]$, there exists a unique $V^{b} \in(0,1-c / 1-\beta)$ that satisfies $V^{b}=\Upsilon_{b}(\cdot)$. (3), (4), and (6) also imply

$$
V^{S}=\frac{\left(1-e^{-\chi_{S}}-x_{s} e^{-x_{S}}\right)(1-c)}{1-\beta}
$$

and this time, the R.H.S. of this equation, denoted by $Y_{s}\left(x_{s}\right)$, is strictly increasing in $x^{s} \in(0, \infty)$ and satisfies: $\Upsilon_{s}(\cdot) \rightarrow 0$ as $x_{s} \rightarrow 0 ; \Upsilon_{s}(\cdot) \rightarrow 1-c / 1-\beta$ as $x_{s} \rightarrow \infty$, thereby there exists a unique solution $V^{s} \in(0,1-c / 1-\beta)$. This completes the proof of Step 2.

\section{Step 3}

Sellers produce a unit for the retail market if $c \leq \bar{c}$, some $\bar{c} \in(0, \beta)$.

Proof of Step 3. Observe that in any given second sub-period, sellers produce a unit for future sale if and only if

$$
c \leq \beta\left(x_{s} \eta\left(x_{s}, 1\right) p_{s}+\left(1-x_{s} \eta\left(x_{s}, 1\right)\right) c\right)
$$

where the R.H.S. represents the expected discounted value of production: the first term in the parenthesis is the expected revenue and the second term is the net value of the produced unit in the next second sub-period - it can be sold to a middleman, which generates $c$ or can be used for saving the production $\operatorname{cost} c$ for the next retail sale. Hence, sellers produce if and only if

$$
c \leq \frac{1-e^{-\chi_{s}}-\chi_{s} e^{-\chi_{s}}}{1-\beta e^{-x_{s}}\left(1+\chi_{s}\right)} \equiv \bar{c} \in(0, \beta) .
$$

This completes the proof of Step 3. 


\section{Proof of Proposition 1}

Differentiation yields

$$
\frac{d p_{i}}{d S}=\frac{\partial \varphi^{i}\left(x_{i}, \cdot\right)}{\partial x_{i}} \frac{d x_{i}}{d S}(1-c),
$$

for $i=s, m$. Remember that $d x_{i} / d S<0, i=s, m$ (see the proof of Step 1 in Theorem 1 ). Below, I show that $\partial \varphi^{i}\left(x_{i}, \cdot\right) / \partial x_{i}>0$ for all the possible values of $x_{i}$. There are three cases.

\section{$\odot$ Case 1. $\boldsymbol{x}_{\boldsymbol{i}}<\boldsymbol{k}_{\boldsymbol{i}}$ :}

Observe that

$$
\frac{\partial \varphi^{i}\left(x_{m}, \cdot\right)}{\partial x_{i}}=-\frac{\frac{k_{i}}{x_{i}}\left(1-\frac{\Gamma\left(k_{i}+1, x_{i}\right)}{\Gamma\left(k_{i}+1\right)}\right)}{\eta(\cdot)^{2}} \frac{\partial \eta(\cdot)}{\partial x_{i}}+\frac{\frac{\partial}{\partial x_{i}}\left[\frac{k_{i}}{x_{i}}\left(1-\frac{\Gamma\left(k_{i}+1, x_{i}\right)}{\Gamma\left(k_{i}+1\right)}\right)\right]}{\eta(\cdot)} .
$$

The first term in the R.H.S. of (11) is positive. The numerator of the second term in (11) is

$$
\frac{\partial}{\partial x_{i}}\left[\frac{k_{i}}{x_{i}}\left(1-\frac{\Gamma\left(k_{i}+1, x_{i}\right)}{\Gamma\left(k_{i}+1\right)}\right)\right]=\frac{\partial}{\partial x_{i}}\left[\sum_{j=k_{i}}^{\infty} \frac{x_{i}^{j} e^{-x_{i}}}{j !} \frac{k_{i}}{j+1}\right]=\sum_{j=k_{i}}^{\infty} \frac{x_{i}^{j-1} e^{-x_{i}}\left(j-x_{i}\right)}{j !} \frac{k_{i}}{j+1}>0
$$

if $x_{i}<k_{i}$.

$\odot$ Case 2. $x_{i} \geq k_{i}$ and $\frac{\Gamma\left(k_{i}, x_{i}\right)}{\Gamma\left(k_{i}\right)} \leq \frac{x_{i}^{k_{i}} e^{-x_{i}}}{\Gamma\left(k_{i}\right)}$ :

Rewrite (11) as

$$
x_{i} \eta(\cdot)^{2} \frac{\partial \varphi^{i}\left(x_{i}, \cdot\right)}{\partial x_{i}}=\frac{x_{i}^{k_{i}} e^{-x_{i}}}{\Gamma\left(k_{i}\right)} \frac{\Gamma\left(k_{i}, x_{i}\right)}{\Gamma\left(k_{i}\right)}+\frac{k_{i}}{x_{i}}\left(1-\frac{\Gamma\left(k_{i}+1, x_{i}\right)}{\Gamma\left(k_{i}+1\right)}\right)\left(\frac{x_{i}^{k_{i}} e^{-x_{i}}}{\Gamma\left(k_{i}\right)}-\frac{\Gamma\left(k_{i}, x_{i}\right)}{\Gamma\left(k_{i}\right)}\right) .
$$

If $\Gamma\left(k_{i}, x_{i}\right) / \Gamma\left(k_{i}\right) \leq x_{i}^{k_{i}} e^{-x_{i}} / \Gamma\left(k_{i}\right)$, then the second term above is positive and so $\partial \varphi^{i}\left(x_{i}, \cdot\right) / \partial x_{i}>0$.

$\odot$ Case 3. $\boldsymbol{x}_{\boldsymbol{i}} \geq \boldsymbol{k}_{\boldsymbol{i}}$ and $\frac{\Gamma\left(k_{i}, x_{i}\right)}{\Gamma\left(k_{i}\right)}>\frac{x_{i}^{k_{i}} e^{-x_{i}}}{\Gamma\left(k_{i}\right)}$ :

Using $\Gamma\left(k_{i}+1, x_{i}\right) / \Gamma\left(k_{i}+1\right)=\Gamma\left(k_{i}, x_{i}\right) / \Gamma\left(k_{i}\right)-x_{i}^{k_{i}} e^{-x_{i}} / \Gamma\left(k_{i}+1\right)$, (11) can be further rearranged to $x_{i} \eta(\cdot)^{2} \partial \varphi^{i}\left(x_{i}, \cdot\right) / \partial x_{i}$ 


$$
\begin{aligned}
& =\frac{x_{i}^{k_{i}} e^{-x_{i}}}{\Gamma\left(k_{i}\right)} \eta\left(x_{i}, k_{i}\right)-\frac{\Gamma\left(k_{i}, x_{i}\right)}{\Gamma\left(k_{i}\right)} \frac{k_{i}}{x_{i}}\left(1-\frac{\Gamma\left(k_{i}, x_{i}\right)}{\Gamma\left(k_{i}\right)}\right)+\frac{x_{i}^{k_{i}-1} e^{-x_{i}}}{\Gamma\left(k_{i}\right)} \frac{\Gamma\left(k_{i}, x_{i}\right)}{\Gamma\left(k_{i}\right)} \\
& =\frac{x_{i}^{k_{i}} e^{-x_{i}}}{\Gamma\left(k_{i}\right)}\left[\left(1+\frac{1}{x_{i}}\right) \frac{\Gamma\left(k_{i}, x_{i}\right)}{\Gamma\left(k_{i}\right)}+\frac{k_{i}}{x_{i}}\left(1-\frac{\Gamma\left(k_{i}+1, x_{i}\right)}{\Gamma\left(k_{i}+1\right)}\right)\right]-\frac{\Gamma\left(k_{i}, x_{i}\right)}{\Gamma\left(k_{i}\right)} \frac{k_{i}}{x_{i}}\left(1-\frac{\Gamma\left(k_{i}, x_{i}\right)}{\Gamma\left(k_{i}\right)}\right) \\
& >\frac{x_{i}^{k_{i}} e^{-x_{i}}}{\Gamma\left(k_{i}\right)}-\frac{\Gamma\left(k_{i}, x_{i}\right)}{\Gamma\left(k_{i}\right)}\left(1-\frac{\Gamma\left(k_{i}, x_{i}\right)}{\Gamma\left(k_{i}\right)}\right),
\end{aligned}
$$

where the last inequality is because it holds that

$$
\begin{aligned}
& \frac{\Gamma\left(k_{i}, x_{i}\right)}{\Gamma\left(k_{i}\right)}\left(1-\frac{k_{i}}{x_{i}}\right)\left(1-\frac{\Gamma\left(k_{i}, x_{i}\right)}{\Gamma\left(k_{i}\right)}\right)>\frac{x_{i}^{k_{i}} e^{-x_{i}}}{\Gamma\left(k_{i}\right)}\left[1-\left(1+\frac{1}{x_{i}}\right) \frac{\Gamma\left(k_{i}, x_{i}\right)}{\Gamma\left(k_{i}\right)}\right. \\
& \left.\quad-\frac{k_{i}}{x_{i}}\left(1-\frac{\Gamma\left(k_{i}+1, x_{i}\right)}{\Gamma\left(k_{i}+1\right)}\right)\right] \\
& \Leftarrow\left(1-\frac{k_{i}}{x_{i}}\right)\left(1-\frac{\Gamma\left(k_{i}, x_{i}\right)}{\Gamma\left(k_{i}\right)}\right)>1-\left(1+\frac{1}{x_{i}}\right) \frac{\Gamma\left(k_{i}, x_{i}\right)}{\Gamma\left(k_{i}\right)}-\frac{k_{i}}{x_{i}}\left(1-\frac{\Gamma\left(k_{i}+1, x_{i}\right)}{\Gamma\left(k_{i}+1\right)}\right) \\
& \Leftarrow \frac{1}{x_{i}}\left(\frac{\Gamma\left(k_{i}, x_{i}\right)}{\Gamma\left(k_{i}\right)}-\frac{x_{i}^{k_{i}} e^{-x_{i}}}{\Gamma\left(k_{i}\right)}\right)>0
\end{aligned}
$$

for $\frac{\Gamma\left(k_{i}, x_{i}\right)}{\Gamma\left(k_{i}\right)}>\frac{x_{i}^{k_{i}} e^{-x_{i}}}{\Gamma\left(k_{i}\right)}$. Now, define

$$
\Phi_{g}(x, k) \equiv \frac{x^{k} e^{-x}}{\Gamma(k)}-\frac{\Gamma(k, x)}{\Gamma(k)}\left(1-\frac{\Gamma(k, x)}{\Gamma(k)}\right)
$$

for $x \geq k \in[1, \infty) \subseteq \mathbf{R}_{+}$. Observe that $\lim _{x \rightarrow \infty} \Phi_{g}(x, k)=0$, and

$$
\frac{\partial \Phi_{g}(x, k)}{\partial x}=\frac{x^{k-1} e^{-x}}{\Gamma(k)}\left(k+1-x-2 \frac{\Gamma(k, x)}{\Gamma(k)}\right) \quad \gtreqless 0 \Leftrightarrow \quad x_{\lessgtr}^{\lessgtr} x^{+}
$$

where $x^{+} \in(k, k+1)$ is a unique solution to $x^{+}=k+1-2 \Gamma\left(k, x^{+}\right) / \Gamma(k)$, hence $\partial \Phi_{g}(x, k) / \partial x>0$ at $x=k$. Therefore, if $\Phi_{g}(k, k)>0$ then $\Phi_{g}(x, k)>0$ for all $x \in[k, \infty)$. To show this corner condition $\Phi_{g}(k, k)>0$ holds true, notice first that

$$
\Phi_{g}(k, k)>\frac{k^{k} e^{-k}}{\Gamma(k)}-\frac{1}{4}
$$

holds true for any $k \in[1, \infty)$. Now, observe that

$$
\frac{d}{d k} \ln \left(\frac{k^{k} e^{-k}}{\Gamma(k)}\right)=\ln (k)-\psi(k)
$$


where $\psi(k)=d \ln \Gamma(k) / d k$ is the Psi (or digamma) function, which has the definiteintegral representation that leads to

$$
\begin{gathered}
\psi(k)=\int_{0}^{\infty}\left(e^{-t}-\frac{1}{(1+t)^{k}}\right) \frac{d t}{t} \\
=\ln k-\frac{1}{2 k}-2 \int_{0}^{\infty} \frac{t d t}{\left(t^{2}+k^{2}\right)\left(e^{2 \pi t}-1\right)}
\end{gathered}
$$

(see, for example, Abramowitz and Stegun (1965) p.259). The last expression leads to

$$
\frac{d}{d k} \ln \left(\frac{k^{k} e^{-k}}{\Gamma(k)}\right)=\frac{1}{2 k}+2 \int_{0}^{\infty} \frac{t d t}{\left(t^{2}+k^{2}\right)\left(e^{2 \pi t}-1\right)}>0,
$$

for all $k \in[1, \infty)$. Since $k^{k} e^{-k} / \Gamma(k)=e^{-1}(\simeq 0.37>1 / 4)$ when $k=1$, this implies that the term $k^{k} e^{-k} / \Gamma(k)$ is greater than $1 / 4$. This further implies $\Phi_{g}(k, k)>0$ for all $k \in[1, \infty)$ and $\Phi_{g}(x, k)>0$ for all $x \in[k, \infty)$. This shows that the R.H.S. of (12) is positive and so $\partial \varphi^{i}\left(x_{i}, \cdot\right) / \partial x_{i}>0$.

The above covers all the possible cases and, therefore, it has been shown that $\partial \varphi^{i}\left(x_{i}, \cdot\right) / \partial x_{i}>0$, for all $x_{i} \in(0, \infty), i=s, m$. The result on parameter $M$ follows from exactly the same procedure.

\section{Proof of Proposition 2}

As given in the test, the retail price differential is

$$
(1-c)^{-1}\left(p_{m}-p_{s}\right)=\varphi^{m}(\cdot)-\varphi^{s}(\cdot)=\frac{\frac{k_{m}}{x_{m}}\left(1-\frac{\Gamma\left(k_{m}+1, x_{m}\right)}{\Gamma\left(k_{m}+1\right)}\right)}{\eta\left(x_{m}, k_{m}\right)}-\frac{\frac{1-e^{-x_{s}}-x_{s} e^{-x_{s}}}{x_{s}}}{\eta\left(x_{s}, 1\right)} .
$$

From this, it follows that $\eta\left(x_{m}, k_{m}\right) \eta\left(x_{s}, 1\right)\left(\varphi^{m}-\varphi^{s}\right)$

$$
\begin{aligned}
= & \frac{1-e^{-x_{s}}}{x_{s}} \frac{k_{m}}{x_{m}}\left(1-\frac{\Gamma\left(k_{m}+1, x_{m}\right)}{\Gamma\left(k_{m}+1\right)}\right)-\left\{\frac{\Gamma\left(k_{m}\right), x_{m}}{\Gamma\left(k_{m}\right)}+\frac{k_{m}}{x_{m}}\left(1-\frac{\Gamma\left(k_{m}+1, x_{m}\right)}{\Gamma\left(k_{m}+1\right)}\right)\right\} \\
& \times \frac{1-e^{-x_{s}}-x_{s} e^{-x_{s}}}{x_{s}} \\
= & {\left[\frac{k_{m}}{x_{m}}\left(1-\frac{\Gamma\left(k_{m}+1, x_{m}\right)}{\Gamma\left(k_{m}+1\right)}\right)-\frac{1-e^{-x_{s}}-x_{s} e^{-x_{s}}}{x_{s}}\right] e^{-x_{s}} } \\
= & {\left[\frac{\left(1-e^{-x_{s}}\right)\left(k_{m} x_{s}-x_{m}\right)}{x_{m} x_{s}}+\frac{\Gamma\left(k_{m}, x_{m}\right)}{\Gamma\left(k_{m}\right)}-\frac{x_{m}^{k_{m}-1} e^{-x_{m}}}{\Gamma\left(k_{m}\right)}\right] e^{-x_{s}} }
\end{aligned}
$$




$$
=\left[\left(1-e^{-x_{s}}\right)\left(\left(M k_{m}+S\right) x_{s}-1\right)+\frac{\Gamma\left(k_{m}-1, \frac{1-S x_{s}}{M}\right)}{\Gamma\left(k_{m}-1\right)} \chi_{s}\left(1-S x_{s}\right)\right] \frac{e^{-x_{s}}}{\left(1-S x_{s}\right) x_{s}}
$$

where I have used (8) for the second equality, (8) and $\Gamma\left(k_{m}+1, x_{m}\right) / \Gamma\left(k_{m}+1\right)=$ $\Gamma\left(k_{m}, x_{m}\right) / \Gamma\left(k_{m}\right)+x_{m}^{k_{m}} e^{-x_{m}} / \Gamma\left(k_{m}+1\right)$ for the third equality, and (1) and $\Gamma\left(k_{m}, x_{m}\right) / \Gamma\left(k_{m}\right)=\Gamma\left(k_{m}-1, x_{m}\right) / \Gamma\left(k_{m}-1\right)+x_{m}^{k_{m}-1} e^{-x_{m}} / \Gamma\left(k_{m}\right)$ for the last equality. Define $\Lambda_{x}\left(x_{s}\right)$ as the parenthesis terms in the last expression above for $x_{s} \in(0,1 / S)$ and $k_{m}>1$. Then, it satisfies $\Lambda_{x}\left(x_{s}\right) \rightarrow 0$ as $x_{s} \rightarrow 0, \Lambda_{x}\left(x_{s}\right) \rightarrow\left(1-e^{-\frac{1}{S}}\right) M k_{m} / S>0$ as $x_{s} \rightarrow 1 / S$, and

$$
\begin{aligned}
& \frac{d \Lambda_{x}\left(x_{s}\right)}{d x_{s}}=\left(M k_{m}+S\right)\left(1-e^{-x_{s}}+x_{s} e^{-\chi_{s}}\right)-\frac{\Gamma\left(k_{m}-1, \frac{1-S x_{s}}{M}\right)}{\Gamma\left(k_{m}-1\right)}\left(2 S x_{s}-1\right) \\
& +\frac{x_{m}^{k_{m}-2} e^{-x_{m}}}{\Gamma(k-1)} \frac{S x_{s}\left(1-S x_{s}\right)}{M} \\
& =M k_{m}\left(1-e^{-\chi_{s}}+\chi_{s} e^{-\chi_{s}}\right)+S\left(1-e^{-\chi_{s}}+k x_{s} e^{-\chi_{s}}\right) \\
& -\frac{\Gamma\left(k_{m}-1, \frac{1-S x_{s}}{M}\right)}{\Gamma\left(k_{m}-1\right)}\left(S x_{s}\left(k_{m}+1\right)-1\right)>M k_{m}\left(1-e^{-x_{s}}+x_{s} e^{-x_{s}}\right) \\
& +S\left(1-e^{-x_{s}}+k x_{s} e^{-x_{s}}\right)-e^{-x_{s}}\left(S x_{s}\left(k_{m}+1\right)-1\right) \\
& =M k_{m}\left(1-e^{-x_{s}}+x_{s} e^{-x_{s}}\right)+S\left(1-e^{-x_{s}}-x_{s} e^{-x_{s}}\right)+e^{-x_{s}}>0 \text {, }
\end{aligned}
$$

where I have used $\Gamma\left(k_{m}-1, x_{m}\right) / \Gamma\left(k_{m}-1\right)<\Gamma\left(k_{m}, x_{m}\right) / \Gamma\left(k_{m}\right)\left(=e^{-x_{s}}\right.$ by (8)) for the second inequality. This implies $\Lambda_{x}\left(x_{s}\right)>0$ for all $x_{\epsilon}(0,1 / S)$ given $k_{m}>1$. Hence, $\varphi^{m}-\varphi^{s}>0$ and so $p_{m}-p_{s}>0$ for all $k_{m}>1$ and $S, M \in(0, \infty)$. Since $p_{m}-p_{s}=0$ when $k_{m}=1$, this proves the claims in the proposition.

\section{Proof of Proposition 3}

$\odot$ Retail price of middlemen $p_{m}$ :

For the expositional ease, let

$$
\nabla_{1} \equiv \frac{x_{m}}{k_{m}} \frac{\Gamma\left(k_{m}, x_{m}\right)}{\Gamma\left(k_{m}\right)} ; \quad \nabla_{2} \equiv 1-\frac{\Gamma\left(k_{m}+1, x_{m}\right)}{\Gamma\left(k_{m}+1\right)} .
$$

Differentiating $p_{m}\left(=\varphi^{m}\left(x_{m}, k_{m}\right)\right)$ with respect to $k_{m} \in[1, \infty) \subset \mathbf{R}_{+}$,

$$
\left(\nabla_{1}+\nabla_{2}\right)^{2} \frac{d \varphi^{m}\left(x_{m}, k_{m}\right)}{d k_{m}}
$$




$$
\begin{gathered}
=\left(\nabla_{1}+\nabla_{2}\right)^{2} \frac{d}{d k_{m}}\left(\frac{\nabla_{2}}{\nabla_{1}+\nabla_{2}}\right) \\
=-\nabla_{1} \frac{\partial \frac{\Gamma\left(k_{m}+1, x_{m}\right)}{\Gamma\left(k_{m}+1\right)}}{\partial k_{m}}+\nabla_{2}\left(\frac{\nabla_{1}}{k_{m}}-\frac{x_{m}}{k_{m}} \frac{\partial \frac{\Gamma\left(k_{m}, x_{m}\right)}{\Gamma\left(k_{m}\right)}}{\partial k_{m}}\right)+\frac{d x_{m}}{d k_{m}}\left(\left(\nabla_{1}+\nabla_{2}\right) \frac{x_{m}^{k_{m}} e^{-x_{m}}}{\Gamma\left(k_{m}+1\right)}-\frac{\nabla_{1} \nabla_{2}}{x_{m}}\right) .
\end{gathered}
$$

In Step 1 in the proof of Theorem 1, it has been shown that

$$
\frac{d x_{m}}{d k_{m}}=\frac{\frac{\partial\left(\Gamma\left(k_{m}, x_{m}\right) / \Gamma\left(k_{m}\right)\right)}{\partial k_{m}}}{\frac{x_{m}^{k_{m}-1} e^{-x_{m}}}{\Gamma\left(k_{m}\right)}+M e^{-x_{s}}},
$$

where, as already mentioned in the text, I used here that $S=1$.

I now evaluate the above derivatives at $k_{m}=1$. Let $x \equiv x_{m}=x_{S}=1 /(M+1) \in(0,1)$ at $k_{m}=1$. Observe that

$$
\begin{aligned}
\frac{\partial\left(\Gamma\left(k_{m}, x\right) / \Gamma\left(k_{m}\right)\right)}{\partial k_{m}} & =\frac{\partial \Gamma\left(k_{m}, x\right) / \partial k_{m}}{\Gamma\left(k_{m}\right)}-\left.\frac{\Gamma\left(k_{m}, x\right)}{\Gamma\left(k_{m}\right)} \frac{\partial \Gamma\left(k_{m}\right) / \partial k_{m}}{\Gamma\left(k_{m}\right)}\right|_{\left.\right|_{k m=1}} \\
& =e^{-x} \ln x+E_{1}(x)+e^{-x} \gamma
\end{aligned}
$$

where in the second equality I have used:

$$
\left.\frac{\partial \Gamma\left(k_{m}, x\right) / \partial k_{m}}{\Gamma\left(k_{m}\right)}\right|_{\left.\right|_{k m=1}}=\left.\frac{\partial \Gamma\left(k_{m}, x\right)}{\partial k_{m}}\right|_{k_{m=1}}=e^{-x} \ln x+E_{1}(x) ;\left.\frac{\partial \Gamma\left(k_{m}\right) / \partial k_{m}}{\Gamma\left(k_{m}\right)}\right|_{k_{m}=1}=-\gamma
$$

(see Geddes, Glasser, Moore, and Scott (1990) for the former, and Abramowitz and Stegun (1965) p.228 for the latter, for example), where

$$
E_{1}(x)=\int_{x}^{\infty} \frac{e^{-t}}{t} d t
$$

is the exponential integral and $y(=0.5772 \ldots)$ is the Euler-Mascheroni constant. Similarly, observe that

$$
\begin{gathered}
\frac{\partial\left(\Gamma\left(k_{m}+1, x\right) / \Gamma\left(k_{m}+1\right)\right)}{\partial k_{m}}=\frac{\partial}{\partial k_{m}}\left(\frac{\Gamma\left(k_{m}, x\right)}{\Gamma\left(k_{m}\right)}+\frac{x^{k_{m}} e^{-x}}{\Gamma\left(k_{m}+1\right)}\right)_{\left.\right|_{k_{m}=1}} \\
=e^{-\chi}(1+x)(\ln x+\gamma)-x e^{-x}+E_{1}(x) .
\end{gathered}
$$

Applying these derivative expressions, and noting $\nabla_{1}=x e^{-x}$ and $\nabla_{2}=1-e^{-x}-x e^{-x}$ when $k_{m}=1$, one obtains

$$
\left.\left(\nabla_{1}+\nabla_{2}\right)^{2} \frac{d \varphi^{m}\left(x, k_{m}\right)}{d k_{m}}\right|_{k_{m}=1}
$$




$$
=-x e^{-x}\left(E_{1}(x)\left(e^{x}-x\right)-1+e^{-x}+\ln x+y\right)+\frac{x-1+e^{-x}}{1+M}\left(E_{1}(x)+e^{-x}(\ln x+y)\right) .
$$

In the above expression, the terms in the first parenthesis, denoted by $\Theta_{1}(x) \equiv E_{1}(x)\left(e^{x}-x\right)-1+e^{-x}+\ln x+\gamma$, satisfy:

$$
\lim _{x \rightarrow 0} \Theta_{1}(x)=\lim _{x \rightarrow 0}\left(E_{1}(x)+\ln x\right)+\gamma=\lim _{x \rightarrow 0} E_{\text {in }}(x)=0,
$$

where I used $\lim _{x \rightarrow 0} E_{1}(x) x=\lim _{x \rightarrow 0} x e^{-x}=0$ (by the l'Hospital rule) in the first equality, and $E_{1}(x)=-\gamma-\ln x+E_{\text {in }}(x)$ in the second equality, where

$$
E_{\text {in }}(x)=\int_{0}^{x}\left(1-e^{-t}\right) \frac{d t}{t}
$$

is the entire function (see footnote 3, p.228 in Abramowitz and Stegun (1965));

$$
\frac{d \Theta_{1}(x)}{d x}=E_{1}(x)\left(e^{x}-1\right)>0 .
$$

Hence, $\Theta_{1}>0$ for all $x \in(0,1]$. The terms in the second bracket, denoted by $\Theta_{2}(x) \equiv E_{1}(x)+e^{-x}(\ln x+y)$, satisfy:

$$
\begin{gathered}
\lim _{x \rightarrow 0} \Theta_{2}(x)=\lim _{x \rightarrow 0}\left(E_{1}(x)+\ln x\right)+\gamma=\lim _{x \rightarrow 0} E_{\text {in }}(x)=0 ; \quad \Theta_{2}(1)=E_{1}(1)+e^{-1} y>1 ; \\
\frac{d \Theta_{2}(x)}{d x}=-e^{-x}(\ln x+\gamma) \gtreqless 0 \Leftrightarrow x \lesseqgtr e^{-\gamma} ; \quad \Theta_{2}\left(e^{-\gamma}\right)=E_{1}\left(e^{-\gamma}\right)>0 .
\end{gathered}
$$

Hence, $\Theta_{2}(x)$ achieves the unique minimum at $x=0$ within $x \in[0,1]$, which equals to zero, thereby $\Theta_{2}(x)>0$ for all $x \in(0,1]$.

Now, since $\Theta_{1}(x)>0, \Theta_{2}(x)>0$ for all $x \in(0,1]$, the condition of price increase is given by $d \varphi^{m}\left(x, k_{m}\right) / d k_{\left.m\right|_{k_{m}=1}}>0 \Leftrightarrow$

$$
M<\frac{\left(x-1+e^{-x}\right) \Theta_{2}(x)-x e^{-x} \Theta_{1}(x)}{x e^{-x} \Theta_{1}(x)} .
$$

In what follows, I identify the values of $x(=1 /(M+1) \epsilon(0,1))$ (and hence $M \in(0, \infty))$ that satisfy the condition of price increase (13). For this purpose, define

$$
\Omega(x) \equiv\left(x-1+e^{-x}\right) \Theta_{2}(x)-e^{-x} \Theta_{1}(x) .
$$

Note the inequality (13) holds true if and only if $\Omega(x)>0 . \Omega(\cdot)$ satisfies: $\lim _{x \rightarrow 0} \Omega(x)=0$;

$$
\Omega(1)=e^{-1}\left(\Theta_{2}(1)-\Theta_{1}(1)\right)=e^{-1}\left[-E_{1}(1)\left(e^{1}-2\right)+\left(1-e^{-1}\right)(1-\gamma)\right]>0
$$


since $E_{1}(1)\left(e^{1}-2\right) \simeq 0.22 \star 0.72 \simeq 0.16<0.27 \simeq\left(1-e^{-1}\right)(1-\gamma)$;

$$
\frac{d \Omega(x)}{d x}=e^{-x} \Theta_{1}(x)+\left(2\left(1-e^{-x}\right)-x\right) e^{-x}(\ln x+y) .
$$

From the last expression, it follows that $\lim _{x \rightarrow 0} d \Omega(x) / d x=\lim _{x \rightarrow 0}\left(2\left(1-e^{-x}\right)-x\right)$ $\ln x=0$ (by using the l'Hospital's rule twice) and $d \Omega(x) / d x>0$ for $x>e^{-\gamma}$. To identify the sign of the derivative for $x \leq e^{-\gamma}$, suppose that $\Omega(x) \geq 0$ for $x \in\left(0, e^{-\gamma}\right]$. Then, it has to hold that $e^{-x} \Theta_{1}(x) \leq\left(x-1+e^{-x}\right) \Theta_{2}(x)$, which further implies

$$
\begin{aligned}
& \frac{d \Omega(x)}{d x} \leq\left(x-1+e^{-x}\right) \Theta_{2}(x)+\left(2\left(1-e^{-x}\right)-x\right) e^{-x}(\ln x+y) \\
& =\left(x-1+e^{-x}\right) E_{1}(x)+\left(1-e^{-x}\right) e^{-x}(\ln x+y) \equiv \Upsilon(x)
\end{aligned}
$$

for $x \in\left(0, e^{-\gamma}\right]$. Observe that $\lim _{x \rightarrow 0} \Upsilon(x)=0$ (by using the l'Hospital's rule thrice on the first term and twice on the second term) and $Y\left(e^{-\gamma}\right)>0$. Further,

$$
\frac{d \Upsilon(x)}{d x}=\left(1-e^{-x}\right) \Theta_{2}(x)-\left(2-3 e^{-x}\right) e^{-x}(\ln x+y)+\frac{e^{-x}\left(2\left(1-e^{-x}\right)-x\right)}{x} \rightarrow-\infty<0
$$

as $_{x \rightarrow 0}$. This implies there exists some $x^{\prime} \in\left(0, e^{-\gamma}\right)$ such that $Y\left(x^{\prime}\right)=0$ and $Y(x)<0$ for $x<x^{\prime}$. The latter further implies $d \Omega(x) / d x<0$ for $x<x^{\prime}$, a contradiction to $d \Omega(x) / d x \geq 0$ (which is implied by $\Omega(x) \geq 0$ and $\lim _{x \rightarrow 0} \Omega(x)=0$ for an interval of $x$ close to 0 ). Hence, we must have $\Omega(x)<0$ for an interval $x$ close to zero. As $\Omega(x)$ is continuous in $x \in(0,1)$ and $\Omega(1)>0$, this implies that there exists some $x^{\star} \in(0,1)$ such that $\Omega\left(x^{\star}\right)=0$ and $\Omega(x)<0$ for $x \in\left(0, x^{\star}\right)$.

Observe now that $\Omega\left(e^{-\gamma}\right)=-\left(2-x-e^{-\chi}-x e^{-x}\right) E_{1}(x)+\left.e^{-x}\left(1-e^{-\chi}\right)\right|_{x=e^{-y} \simeq 0.56} \simeq$ $-0.55 \star 0.49+0.25<0$. This implies, since $\Omega(x)$ is increasing in $x \in\left(e^{-\gamma}, 1\right)$, it has to be that $x^{\star} \in\left(e^{-\gamma}, 1\right)$. This further implies that $\Omega(x)$ must cross the horizontal axis (of $\Omega(\cdot)=0)$ from below and only once at $x^{\star} \in\left(e^{-\gamma}, 1\right)$. As $\lim _{x \rightarrow 0} \Omega(x)=0<\Omega(1)$, it should hold that

$$
\Omega(x) \leq 0 \text { for } x \leq x^{*} \in(0,1) \text { and } \Omega(x)>0 \text { for } x>x^{*} .
$$

Therefore, the condition of price increase (13) holds true if and only if $x \in\left(x^{\star}, 1\right)$, and since $x=X$ when $k_{m}=1$, this proves the first claim in the proposition with $x^{\star}=X^{\star} \in(0,1)$.

To prove the second claim, it is sufficient to observe that since $x_{m} \rightarrow 1 / M$, $x_{m} \eta\left(x_{m}, k_{m}\right) \rightarrow 1 / M, k_{m} \nabla_{2} \rightarrow 0$ as $k_{m} \rightarrow \infty$, it holds that $\varphi^{m}\left(x_{m}, k_{m}\right) \rightarrow 0$ as $k_{m} \rightarrow \infty$. 


\section{$\odot$ Retail price of sellers $\boldsymbol{p}_{\boldsymbol{s}}$ :}

It is sufficient to observe that $x_{s}(\cdot)$ is strictly decreasing in all $k_{m} \geq 1$ (as shown in Step 1 in the proof of Theorem 1$)$ and $p_{s}\left(=\varphi^{S}(\cdot)\right)$ is strictly increasing in all $x_{s} \in(0,1)$ (as shown in the proof of Proposition 1). In the limit as $k_{m} \rightarrow \infty$, we have $x_{s} \rightarrow 0$ and so $\varphi^{S}\left(x_{s}, 1\right) \rightarrow 0$.

\section{$\odot$ Retail market premium $\boldsymbol{p}_{\boldsymbol{m}}-\boldsymbol{p}_{\boldsymbol{s}}$ :}

The above analysis shows that $p_{m} \rightarrow c$ as $k_{m} \rightarrow \infty$ and $p_{s} \rightarrow c$ as $k_{m} \rightarrow \infty$. Hence, $p_{m}-p_{s} \rightarrow 0$ as $k_{m} \rightarrow \infty$. Since $p_{m}=p_{s}$ when $k_{m}=1$ and $p_{m}>p_{s}$ when $1<k_{m}<\infty$, this implies the price differential must be increasing (decreasing) in low (high) $k_{m}$.

\section{Proof of Proposition 4}

\section{$\odot$ Retail price of middlemen $\boldsymbol{p}_{\boldsymbol{m}}$ :}

With the fixed total supply of middlemen $G=M k_{m}$, the only modification appears in the adding-up restriction (1), which now becomes (with applying $S=1$ )

$$
\frac{G}{k_{m}} x_{m}+x_{s}=1 \text {. }
$$

This affects the analysis in Step 1 in the proof of Theorem 1, so that now I have

$$
\frac{d x_{m}}{d k_{m}}=\frac{\frac{\partial\left(\Gamma\left(k_{m}, x_{m}\right) / \Gamma\left(k_{m}\right)\right)}{\partial k_{m}}+\frac{G x_{m}}{k_{m}^{2}} e^{-\chi_{s}}}{\frac{x_{m}^{k_{m}-1} e^{-x_{m}}}{\Gamma\left(k_{m}\right)}+\frac{G}{k_{m}} e^{-\chi_{s}}} .
$$

Observe that there is an additional, positive term in the numerator of this expression. This modification further affects the following parts of the analysis: the derivative in question becomes

$$
\begin{aligned}
& \left.\left(\nabla_{1}+\nabla_{2}\right)^{2} \frac{d \varphi^{m}\left(x, k_{m}\right)}{d k_{m}}\right|_{\left.\right|_{m=1 \& G=M k_{m}}} \\
& =-x e^{-x}\left(E_{1}(x)\left(e^{x}-x\right)-1+e^{-x}+\ln x+y\right)+\frac{x-1+e^{-\chi}}{1+G}\left(E_{1}(x)+e^{-x}(\ln x+y)\right. \\
& \left.+G x e^{-x}\right),
\end{aligned}
$$

where a positive term is added inside the second bracket; the condition for price increase (13) is then modified to $d \varphi^{m}\left(x, k_{m}\right) / d k_{\left.m\right|_{k_{m}=1 \& G=M k_{m}}}>0 \Leftrightarrow$ 


$$
G\left(1-\frac{x-\left(1-e^{-x}\right)}{\Theta_{1}(x)}\right)<\frac{\left(x-1+e^{-x}\right) \Theta_{2}(x)-x e^{-x} \Theta_{1}(x)}{x e^{-x} \Theta_{1}(x)} .
$$

Observe here that the R.H.S. remains the same as before, while the L.H.S. is now multiplies by a new term which is less than one. As $G=M$ when $k_{m}=1$, this implies that the above inequality holds for all $x=X>x^{\star}=X^{\star} \in(0,1)$ (see the proof of Proposition 3) and so $d \varphi^{m}\left(x, k_{m}\right) / d k_{\left.m\right|_{k_{m}=1 \& G=M k_{m}}}>0$ for all $x \in\left(\chi^{\star}, 1\right)$. This proves the first claim for $p_{m}$ in the proposition.

The second claim can be shown by using the following property (see Temme (1996) p.285):

$$
\frac{\Gamma\left(k_{m}, x_{m}\right)}{\Gamma\left(k_{m}\right)} \rightarrow D \text { as } k_{m} \rightarrow \infty
$$

where $D \in[0,1]$ satisfies: $D=1$ if and only if $x_{m}<k_{m} ; D=0$ if and only if $x_{m}>k_{m}$.

Throughout the proof given below, keep in mind that with the fixed total supply $G=M k_{m} \in(0, \infty)$, it has to be that $M=G / k_{m} \rightarrow 0$ as $k_{m} \rightarrow \infty$, thus $x_{m} \rightarrow \infty$ as $k_{m} \rightarrow \infty$. There are three cases. Consider first the case $G<1$. Suppose $x_{m}>k_{m}$ as $k_{m} \rightarrow \infty$. This leads to $\Gamma\left(k_{m}, x_{m}\right) / \Gamma\left(k_{m}\right) \rightarrow 0$ as $k_{m} \rightarrow \infty$ by (14) and so $x_{s} \rightarrow \infty$ as $k_{m} \rightarrow \infty$ by (8). However, this contradicts to (1) which requires $x_{s} \in[0,1]$. Suppose $x_{m}<k_{m}$ as $k_{m} \rightarrow \infty$. Then, $\Gamma\left(k_{m}, x_{m}\right) / \Gamma\left(k_{m}\right) \rightarrow 1$ as $k_{m} \rightarrow \infty$ by (14) and so $x_{s} \rightarrow 0$ as $k_{m} \rightarrow \infty$ by (8). However, this contradicts to (1) and $G<1$, or

$$
M\left(x_{m}-k_{m}\right)+x_{s}=1-G>0
$$

which requires $x_{s}>0$, if $x_{m}<k_{m}$. Therefore, the only possible solution when $G<1$ is $x_{m}=k_{m}$ as $k_{m} \rightarrow \infty$, which in turn leads to $x_{s}=1-G$ by (1), as is consistent with (14), requiring $\Gamma\left(k_{m}, x_{m}\right) / \Gamma\left(k_{m}\right)=e^{-x_{s}} \in(0,1)$ as $k_{m} \rightarrow \infty$ and $x_{m}=k_{m}$. In this solution, it holds that:

$$
\eta\left(x_{m}, k_{m}\right)=\frac{\Gamma\left(k_{m}, x_{m}\right)}{\Gamma\left(k_{m}\right)}+\frac{k_{m}}{x_{m}}\left(1-\frac{\Gamma\left(k_{m}, x_{m}\right)}{\Gamma\left(k_{m}\right)}\right)-\frac{x^{k_{m}-1} e^{-x}}{\Gamma\left(k_{m}\right)} \rightarrow 1 \text { as } k_{m} \rightarrow \infty
$$

because $x^{k_{m}-1} e^{-x} / \Gamma\left(k_{m}\right) \rightarrow 0$ as $k_{m} \rightarrow \infty$ for any $x_{m} / k_{m} \in(0, \infty)$;

$$
\varphi^{m}\left(x_{m}, k_{m}\right) \rightarrow 1-e^{-(1-G)} \quad \text { as } \quad k_{m} \rightarrow \infty .
$$

Consider next the case $G=1$. Suppose $x_{m}<k_{m}$ as $k_{m} \rightarrow \infty$. Then, $\Gamma\left(k_{m}, x_{m}\right) / \Gamma\left(k_{m}\right) \rightarrow 1$ as $k_{m} \rightarrow \infty$ by (14) and so $x_{s} \rightarrow 0$ as $k_{m} \rightarrow \infty$ by (8). However, this contradicts to (1) and $G=1$, or

$$
M\left(x_{m}-k_{m}\right)+x_{s}=1-G=0
$$


which requires $x_{s}>0$, if $x_{m}<k_{m}$. Similarly, $x_{m} \geq k_{m}$ as $k_{m} \rightarrow \infty$ cannot be the solution. Therefore, there is no limiting solution as $k_{m} \rightarrow \infty$ with the fixed total supply $G=M k_{m}$ when $G=1$.

Consider finally the case $G>1$. Then, by (1),

$$
M\left(x_{m}-k_{m}\right)+x_{s}=1-G<0,
$$

implying that $x_{m}<k_{m}$ as $k_{m} \rightarrow \infty$, leading to $\Gamma\left(k_{m}, x_{m}\right) / \Gamma\left(k_{m}\right) \rightarrow 1$ and $x_{s} \rightarrow 0$ by (8) and (14), is the only solution. Therefore, $1-\Gamma\left(k_{m}, x_{m}\right) / \Gamma\left(k_{m}\right) \rightarrow 0$ as $k_{m} \rightarrow \infty$, which implies $\varphi^{m}(\cdot) \rightarrow 0$ as $k_{m} \rightarrow \infty$ and thus $\varphi^{m}(\cdot)>\lim _{k_{m} \rightarrow \infty} \varphi^{m}(\cdot)$ for all $k_{m} \geq 1$.

Therefore, it has to hold that $p_{m}>c$ as $k_{m} \rightarrow \infty$ if and only if $G<1$ or $X=1 / G+1>X^{\star \star}=1 / 2$.

\section{$\odot$ Retail market premium $\boldsymbol{p}_{\boldsymbol{m}}-\boldsymbol{p}_{\boldsymbol{s}}$ :}

With fixed $G=M k_{m}$, we have

$$
\frac{d x_{s}}{d k_{m}}=-\frac{\frac{\partial\left(\Gamma\left(k_{m}, x_{m}\right) / \Gamma\left(k_{m}\right)\right)}{\partial k_{m}}-\frac{x_{m}^{k_{m}-1} e^{-x_{m}}}{\Gamma\left(k_{m}\right)} \frac{1-\chi_{s}}{G}}{\frac{x_{m}^{k_{m}-1} e^{-x_{m}}}{\Gamma\left(k_{m}\right)} \frac{k_{m}}{G}+e^{-\chi_{s}}}=-\left.\frac{E_{1}(x)+e^{-\chi}(\ln x+\gamma)-e^{-\chi \chi 1-\chi} \frac{1-}{M}}{e^{x}\left(1+\frac{1}{M}\right)}\right|_{k_{m}=1 \& G=M k_{m}},
$$

and

$$
\begin{aligned}
& \left(\nabla_{1}+\nabla_{2}\right)^{2}\left(\frac{d \varphi^{m}\left(x_{m}, k_{m}\right)}{d k_{m}}-\frac{d \varphi^{s}\left(x_{s}, 1\right)}{d k_{m}}\right)_{\left.\right|_{k_{m}=1} \& G=M k_{m}} \\
& =-\chi e^{-\chi} \Theta_{1}(x)+\left(x-1+e^{-\chi}\right) \Theta_{2}(x)-(1-\chi) \chi e^{-\chi}
\end{aligned}
$$

where, as before, $\Theta_{1}(x) \equiv E_{1}(x)\left(e^{x}-x\right)-1+e^{-x}+\ln x+y>0$ and $\Theta_{2}(x) \equiv E_{1}(x)+$ $e^{-x}(\ln x+y)>0$. Define the L.H.S. of the above derivative as

$$
\Omega_{f}(x) \equiv E_{1}(x)\left(-\left(1-e^{-x}\right)+x^{2} e^{-x}\right)+x e^{-x}\left(x-e^{-x}\right)-\left(1-e^{-x}\right) e^{-x}(\ln x+y) .
$$

$\Omega f($.$) satisfies: \lim _{x \rightarrow 0} \Omega_{f}(x)=0 ; \Omega_{f}(1)=-E_{1}(1)\left(1-2 e^{-1}\right)+e^{-1}\left(1-e^{-1}\right)(1-\gamma)=$ $-0.22 \star 0.26+0.23 \star 0.43>0$;

$$
\frac{d \Omega_{f}(x)}{d x}=-E_{1}(x) e^{-x}(1-x)^{2}+e^{-x}\left(x\left(2-x+e^{-x}\right)-e^{-x}\right)+\left(1-2 e^{-x}\right) e^{-x}(\ln x+y) .
$$

Note $d \Omega_{f}(x) / d x=e^{-1}+\left(1-2 e^{-1}\right) y>0$ at $x=1$.

Now, suppose that there exists some interval of $x$ in the neighborhood of $x=0$ such that $\Omega_{f}(x) \leq 0$. Then, within that interval of $x$, we must have

$$
e^{-x}(\ln x+y) \geq \frac{1}{1-e^{x}}\left[x e^{-x}-\frac{1-e^{-x}-x^{2} e-x}{1-e^{-x}} E_{1}(x)\right] .
$$


Applying this inequality to the expression of $d \Omega_{f}(x) / d x$, we have

$$
\begin{gathered}
\frac{d \Omega_{f}(x)}{d x} \geq \frac{1-e^{-x}-x e^{-x}}{1-e^{-x}}\left[e^{-x}\left(x-e^{-x}\right)+\frac{x e^{-x}\left(1-e^{-x}\right)}{1-e^{-x}-x e^{-x}}-\left(1-e^{-x}-x e^{-x}\right) E_{1}(x)\right] \\
\equiv \frac{1-e^{-x}-x e^{-x}}{1-e^{-x}} \Upsilon_{f}(x) .
\end{gathered}
$$

Observe that $\Upsilon_{f}(x) \rightarrow 1>0$ as $x \rightarrow 0$. This implies that $d \Omega_{f}(x) / d x \geq 0$ for an interval close to zero, if $\Omega_{f}(x) \leq 0$. However, this is impossible since $\Omega_{f}(x) \rightarrow 0$ as $x \rightarrow 0$. Hence, we must have $\Omega_{f}(x)>0$ in the interval close to $x=0$.

This result further implies that if $\Omega_{f}(x)<0$ in some interval of $x \in(0,1)$ then, since $\Omega_{f}(1)>0$, there must exist at least two points, denoted $x_{-}>x_{+} \in(0,1)$, such that $d \Omega_{f}(x) / d x=0$ at $x=x_{-}, x_{+}$and $\Omega_{f}\left(x_{-}\right)<0<\Omega_{f}\left(x_{+}\right)$. Keeping this in mind, suppose that there exists some $x_{\star} \in(0,1)$ such that $d \Omega_{f}(x) / d x=0$ at $x=\chi_{\star}$ (if not, then the claim holds true automatically). Then, we must have

$$
\begin{aligned}
\Omega_{f}\left(x_{\star}\right)= & \frac{1-e^{-\chi_{\star}}-\chi_{\star} e^{-\chi_{\star}}}{1-2 e^{-\chi_{\star}}}\left[-\left(1-e^{-\chi_{\star}}-\chi_{\star} e^{-\chi_{\star}}\right) E_{1}\left(x_{\star}\right)\right. \\
& \left.+e^{-\chi_{\star}}\left(x_{\star}-e^{-\chi_{\star}}+\frac{\chi_{\star}\left(1-e^{-\chi_{\star}}\right)}{1-e^{-\chi_{\star}}-\chi_{\star} e^{-\chi_{\star}}}\right)\right] .
\end{aligned}
$$

Here, the terms in the parenthesis satisfy

$$
\begin{aligned}
f_{f}(x) & \equiv-\left(1-e^{-x}-x e^{-x}\right) E_{1}(x)+e^{-x}\left(x-e^{-x}+\frac{x\left(1-e^{-x}\right)}{1-e^{-x}-x e^{-x}}\right) \\
& >-\left(1-e^{-x}-x e^{-x}\right) E_{1}(x)+e^{-x}\left(1-e^{-x}\right) \equiv f_{r}(x)>0
\end{aligned}
$$

for all $x \in(0,1]$, where $f_{r}(x)>0$ was introduced in the proof of Proposition 3. Hence, for all $x \in(0,1]$, it holds that $f_{f}(x)>0$, and the result obtained there applies: since $1-e^{-x}-x e^{-x}>0$ for all $x \in(0,1]$ and $1-2 e^{-x}>0$ if and only if $x>\ln (2) \in(0,1)$, we must have $\Omega_{f}\left(x_{\star_{-}}\right)<0<\Omega_{f}\left(x_{\star_{+}}\right)$for some $x_{\star_{-}}<\ln (2)<\chi_{\star_{+}}$if $d \Omega_{f}(x) / d x=0$ at $x=\chi_{\star_{-}}, \chi_{\star_{+}}$, and so it is impossible to have $x_{-}>x_{+} \in(0,1)$ satisfying $d \Omega_{f}(x) / d x=0$ at $x=x_{-}, x_{+}$and $\Omega_{f}\left(x_{-}\right)<0<\Omega_{f}\left(x_{+}\right)$.

All in all, the above covers all the possibilities of $\Omega_{f}(x) \leq 0$ for $x \in(0,1]$, which turns out to be impossible, and so we must have $\Omega_{f}(x)>0$ for all $x \in(0,1]$. This proves the first claim.

The second claim follows from the result obtained in the proof of $p_{m}$ above: when $G<1$, we have

$$
\varphi^{m}\left(x_{m}, k_{m}\right)-\varphi^{s}\left(x_{s}, 1\right) \rightarrow \frac{e^{-x_{s}}\left(x_{s}-1+e^{-x_{s}}\right)}{1-e^{x_{s}}}>0
$$

where $x s \rightarrow 1-G>0$, as $k_{m} \rightarrow \infty$; when $G>1$, 


$$
\varphi^{m}\left(x_{m}, k_{m}\right)-\varphi^{s}\left(x_{s}, 1\right) \rightarrow 0 .
$$

as $k_{m} \rightarrow \infty$. Therefore, $p_{m}-p_{s}>0$ as $k_{m} \rightarrow \infty$ if and only if $G<1$ or $X=1 / G+1>X^{\star \star}=1 / 2$.

\section{Proof of Theorem 2}

From the free entry condition (9), the fixed point condition for the equilibrium number of middlemen $M \in(0, \infty)$ is given by

$$
\Phi_{m}(M, \cdot) \equiv 1-\frac{\Gamma\left(k_{m}+1, x_{m}\right)}{\Gamma\left(k_{m}+1\right)}=\frac{c(1-\beta)+c_{k}}{1-c} \equiv C
$$

where $x_{m}=x_{m}(M)$ is strictly decreasing in $M$ and satisfies $x_{m} \rightarrow 0$ as $M \rightarrow \infty$, as shown in the proof of Theorem 1. It then follows that $\Phi_{m}=\Phi_{m}(M, \cdot)$ is continuous and strictly decreasing in $M \in(0, \infty)$ and satisfies $\Phi_{m} \rightarrow 0<C$ as $M \rightarrow \infty$. Therefore, with $\bar{C} \equiv \lim _{M \rightarrow 0} \Phi_{m} \in(0,1)$, there exists a unique $M \in(0, \infty)$ that satisfies (15) given $C \in(0, \bar{C})$. The comparative statistics of $C$ is immediate: the equilibrium $M$ is strictly decreasing in $C$ satisfying $M \rightarrow \infty$ as $C \rightarrow 0$ and $M \rightarrow 0$ as $C \rightarrow \bar{C}$.

For the comparative statics of $k_{m}$, observe that

$$
\frac{d \Phi_{m}}{d k_{m}}=-\frac{\partial}{\partial k_{m}} \frac{\Gamma\left(k_{m}+1, x_{m}\right)}{\Gamma\left(k_{m}+1\right)}+\frac{x_{m}^{k_{m}} e^{-x_{m}}}{\Gamma\left(k_{m}+1\right)} \frac{d x_{m}}{d k_{m}} .
$$

Evaluating this derivative at $k_{m}=1$ using the expression of $\left.\frac{\partial}{\partial k_{m}} \frac{\Gamma\left(k_{m}+1, x_{m}\right)}{\Gamma\left(k_{m}+1\right)}\right|_{k_{m}=1}$ and $\left.\frac{d x_{m}}{d k_{m}}\right|_{k_{m}=1}$ derived in the proof of Proposition 3, we have

$$
\frac{d \Phi_{m}}{\left.d k_{m}\right|_{k_{m}=1}}=-\left(1-x^{2}\right) E_{1}(x)+x e^{-x}-\left(1+x-x^{2}\right) e^{-x}(\ln x+y) \equiv \Omega_{m}(x) .
$$

Observe that: $\lim _{x \rightarrow 0} \Omega_{m}(x)=-\lim _{x \rightarrow 0}\left(E_{1}(x)+e^{-x}(\ln (x)+\gamma)\right)=0 ; \Omega_{m}(1)=$ $e^{-1}(1-y)>0$

$$
\frac{d \Omega_{m}(x)}{d x}=x\left[2 E_{1}(x)-e^{-x}+(3-x) e^{-x}(\ln x+y)\right] \equiv x f_{m}(x) .
$$

Here, the terms in the parenthesis satisfy $f_{m}(x) \rightarrow-\infty<0$ as $x \rightarrow 0$ and $0<2 E_{1}(1)+e^{-1}(2 y-1)=f_{m}(1)$, implying that there exists some $x_{. \star} \in(0,1)$ such that $f_{m}\left(x_{. \star}\right) \geq 0$ if and only if $x \geq x_{. \star}$. Since $\Omega_{m}(0)=0<\Omega_{m}(1)$, this implies $\Omega_{m}(x) \geq 0$ if and only if $x \geq x_{\text {.*. }}$ As $\Phi_{m}(M)$ is monotone decreasing in $M$, the last result implies that $M$ is decreasing in low $k_{m}$ if $x=1 / M+1<x_{\star \star}$ and is increasing in low $k_{m}$ if $x=1 / M+1>x_{. \star}$. As $M$ is strictly decreasing in $c, c_{k}$, this proves the claim in the proposition. 
As for large $k_{m}$, notice that the L.H.S of the fixed point condition (15) should be a positive number less than one (given $C \in(0, \bar{C})<1)$. This is the case if and only if $x_{m}=k_{m}$ and $x_{s}=1-M k_{m}>0$ as $k_{m} \rightarrow \infty$ (see the property (14) in the proof of Proposition 4). This is possible only when $M \rightarrow 0$ as $k_{m} \rightarrow \infty$.

\section{Proof of Proposition 5}

\section{$\odot$ Retail price of middlemen $\boldsymbol{p}_{\boldsymbol{m}}$ :}

Applying the free entry condition (15) to the retail price of middlemen (3), $i=m$, I get

$$
\frac{p_{m}-c}{1-c}=\varphi^{m}\left(x_{m}, k_{m}\right)=\frac{C}{\frac{x_{m}}{k_{m}} \frac{\Gamma\left(k_{m}, x_{m}\right)}{\Gamma\left(k_{m}\right)}+C} .
$$

Since $C$ is constant, the behavior of the price is dictated by that of the first term in the denominator of the R.H.S., denoted by

$$
B\left(x_{m}, k_{m}\right) \equiv \frac{x_{m}}{k_{m}} \frac{\Gamma\left(k_{m}, x_{m}\right)}{\Gamma\left(k_{m}\right)},
$$

where $x_{m}=x_{m}\left(k_{m}, M\right)$ and $M=M\left(k_{m}\right)$ is determined by the free entry condition (15). Observe that

$$
\frac{d B\left(x_{m}, k_{m}\right)}{d k_{m}}=\frac{\partial B\left(x_{m}, k_{m}\right)}{\partial k_{m}}+\frac{d x_{m}}{d k_{m}}\left(\frac{1}{k_{m}} \frac{\Gamma\left(k_{m}, x_{m}\right)}{\Gamma\left(k_{m}\right)}-\frac{x_{m}^{k_{m}} e^{-x_{m}}}{\Gamma\left(k_{m}+1\right)}\right) .
$$

To compute the term $\frac{d x_{m}}{d k_{m}}=\frac{d x_{m}}{d k_{m}}+\frac{\partial x_{m}}{\partial M} \frac{d M}{d k_{m}}$, observe from the proof of Theorem 2 that

$$
\frac{d M}{d k_{m}}=\frac{\frac{\partial\left(\Gamma\left(k_{m}+1, x_{m}\right) / \Gamma\left(k_{m}+1\right)\right)}{\partial k_{m}}+\frac{x_{m}^{k_{m}} e^{-x_{m}}}{\Gamma\left(k_{m}+1\right)} \frac{\partial x_{m}}{\partial k_{m}}}{\frac{x_{m}^{k_{m}} e^{-x_{m}}}{\Gamma\left(k_{m}+1\right)} \frac{\partial x_{m}}{\partial M}}
$$

and from the proof of Theorem 1 that

$$
\frac{\partial x_{m}}{\partial k_{m}}=\frac{\frac{\partial\left(\Gamma\left(k_{m}, x_{m}\right) / \Gamma\left(k_{m}\right)\right)}{\partial k_{m}}}{\frac{x_{m}^{k_{m}-1} e^{-x_{m}}}{\Gamma\left(k_{m}\right)}+M e^{-\chi_{s}}}, \quad \frac{\partial x_{m}}{\partial M}=-\frac{x_{m} e^{-\chi_{s}}}{\frac{x_{m}^{k_{m}-1} e^{-x_{m}}}{\Gamma\left(k_{m}\right)}+M e^{-x_{s}}} .
$$

Evaluating these derivatives at $k_{m}=1$, we get

$$
\left.\frac{d B\left(x, k_{m}\right)}{d k_{m}}\right|_{k_{m}=1}=\frac{1}{\chi}\left[\left(x^{2}-x+1\right) E_{1}(x)+e^{-x}(\ln x+y)-x e^{-x}\right] \equiv \frac{1}{\chi} \Omega_{r}(x) .
$$

Observe that: $\Omega_{r}(x) \rightarrow 0$ as $x \rightarrow 0 ; \Omega_{r}(1)=-e^{-1}(1-\gamma)+E_{1}(1) \simeq-0.37 \star(1-0.57)+$ $0.22>0 ; d \Omega_{r}(x) / d x=-e^{-x}(\ln x+\gamma)+(2 x-1) E_{1}(x)$. Here, $d \Omega_{r}(x) / d x / x=2 E_{1}(x)-$ 
$e^{-x}(\ln x+\gamma)+E_{1}(x) / x=2 E_{1}(x)-E_{\text {in }}(x) / x \rightarrow+\infty$ as $x \rightarrow 0$ (since $\lim _{x \rightarrow 0} E_{\text {in }}(x) / x=$ $\lim _{x \rightarrow 0} 1-e^{-x} / x=1$ by the l'Hospital rule) and $\frac{d \Omega_{r}(x)}{d x} /\left.x\right|_{x=1}=-e^{-1} \gamma+E_{1}(1) \simeq$ $-0.37 \star 0.57+0.22>0$. Hence, if there is no $x \in(0,1)$ such that $d \Omega_{r}(x) / d x=0$ then $\Omega_{r}(x)>0$ for all $x \in(0,1]$. If there is some $\breve{x} \in(0,1)$ such that $d \Omega_{r}(x) / d x=0$ at $x=\breve{x}$ then observe that

$$
\left.\Omega_{r}(\breve{x})=\breve{x}\left[E_{1}(\breve{x})(1+\breve{x})-e^{-\check{x}}\right]\right]=\breve{x} \Xi_{r}(\breve{x})>0
$$

since $\Xi_{r}(\breve{x}) \rightarrow+\infty$ as $\breve{x} \rightarrow 0, \quad \Xi_{r}(1)=2 E_{1}(1)-e^{-1} \simeq 2^{\star} 0.22-0.37>0, \quad \frac{d \Xi_{r}(\check{x})}{d x}=$ $E_{1}(\breve{x})-\frac{e^{-\breve{x}}}{\check{x}} \rightarrow-\infty$ as $\breve{x} \rightarrow 0, \frac{d \Xi_{r}(\check{x})}{d \check{x}} \rightarrow E_{1}(1)-e-1 \simeq 0.22-0.37<0$, as $\breve{x} \rightarrow 1$ and $\frac{d^{2} \Xi_{r}(\check{x})}{d \check{x}^{2}}=\frac{e^{-\tilde{x}}}{\check{x}^{2}}>0$ - the latter three properties imply $\frac{d \Xi_{r}(\check{x})}{d \ddot{x}}<0$ for all $\breve{x} \in(0,1)$ and so all in all $\Xi_{r}(\breve{x})>0$ for all $\breve{x} \in(0,1]$. Therefore, we must have $\Omega_{r}(x)>0$, and so $\frac{d B\left(x, k_{m}\right)}{d k_{m}}>0$, for all $x \in(0,1]$, proving that $p_{m}$ is decreasing in small $k_{m}$.

For large values of $k_{m}$, observe that $\frac{\Gamma\left(k_{m}+1, x_{m}\right)}{\Gamma\left(k_{m}+1\right)} \rightarrow \frac{\Gamma\left(k_{m}, x_{m}\right)}{\Gamma\left(k_{m}\right)}$ as $k_{m} \rightarrow \infty$. From the property (14) and the free entry condition (15), we must have $x_{m}=k_{m}$ and $\eta\left(x_{m}, k_{m}\right) \rightarrow 1$ as $k_{m} \rightarrow \infty$. This implies that denominator of $\varphi^{m}$, which is $x_{m} \eta\left(x_{m}, k_{m}\right) / k_{m}$, approaches to one (the highest possible value) as $k_{m} \rightarrow \infty$. Since the numerator of $\varphi^{m}$ is constant, this implies that $p_{m}$ approaches to a lowest possible value as $k_{m} \rightarrow \infty$.

\section{$\odot$ Retail market premium $\boldsymbol{p}_{\boldsymbol{m}}-\boldsymbol{p}_{\boldsymbol{s}}$ :}

With free entry, using the derivative expressions derived above, we have

$$
\frac{d x_{s}}{d k_{m}}=\frac{\partial x_{s}}{\partial k_{m}}+\frac{\partial x_{s}}{\partial M} \frac{d M}{d k_{m}}=-\left.\frac{1}{x e^{-\chi}}\left[\chi e^{-\chi}-e^{-\chi}(\ln x+\gamma)-E_{1}(x)(1-x)\right]\right|_{k_{m}=1},
$$

and

$$
\begin{gathered}
\left(\nabla_{1}+\nabla_{2}\right)^{2}\left(\frac{d \varphi^{m}\left(x, k_{m}\right)}{d k_{m}}-\frac{d \varphi^{s}(x, 1)}{d k_{m}}\right)_{\left.\right|_{k_{m}=1}}=x^{2} e^{-x} E_{1}(x)+\left(1-e^{-\chi}\right)\left[x e^{-\chi}-e^{-x}(\ln x+\gamma)\right. \\
\left.-E_{1}(x)\right] \\
>\left(1-e^{-x}\right)\left[x e^{-x}-e^{-x}(\ln x+\gamma)-\left(1-x e^{-x}\right) E_{1}(x)\right] \\
\equiv\left(1-e^{-x}\right) \Omega_{s}(x) .
\end{gathered}
$$

Observe that: $\Omega_{s}(x) \rightarrow 0$ as $x \rightarrow 0 ; \Omega_{S}(1)=e^{-1}(1-\gamma)-\left(1-e^{-1}\right) E_{1}(1) \simeq$ $0.37 \star(1-0.57)-(1-0.37)^{\star} 0.22>0 ; \frac{d \Omega_{s}(x)}{d x}=\left(1-x-e^{-x}\right) e^{-x}+e^{-x}(\ln x+y)+(1-x)$ $e^{-x} E_{1}(x)$. Here, $\frac{d \Omega_{s}(x)}{d x} / x \rightarrow \frac{1-x-e^{-x}}{x}+\frac{\ln x+y+E_{1}(x)}{x} \rightarrow 1$ as $x \rightarrow 0$ by the l'Hospital rule, and $\frac{d \Omega_{s}(x)}{d x} /\left.x\right|_{x=1}=e^{-1}\left(y-e^{-1}\right) \simeq 0.37 \star(0.57-0.37)>0$. Hence, if there is no $x \in(0,1)$ 
such that $d \Omega_{S}(x) / d x=0$ then $\Omega_{s}(x)>0$ for all $x \in(0,1]$. If there is some $\check{x} \in(0,1)$ such that $d \Omega_{S}(x) / d x=0$ at $x=\breve{x}$ then observe that

$$
\left.\frac{d^{2} \Omega_{S}(x)}{d x^{2}}\right|_{x=\check{x}}=-e^{-\check{x}}\left[\frac{\check{x}-1+e^{-\check{x}}}{\check{x}}+E_{1}(\check{x})\right]<0,
$$

implying that it is impossible to have some $x \in(0,1)$ such that $\Omega_{s}(x) \leq 0$. Therefore, $\Omega_{s}(x)>0$ for all $x \in(0,1]$, proving the first claim on low $k_{m}$.

To prove the second claim on large $k_{m}$, observe that $x_{m}=k_{m}$ as $k_{m} \rightarrow \infty$ and, together with the free entry condition (15), $\Gamma\left(k_{m}, x_{m}\right) / \Gamma\left(k_{m}\right)=e^{-x_{s}} \rightarrow 1-C$ as $k_{m} \rightarrow \infty$ imply that

$$
(1-c)^{-1}\left(p_{m}-p_{s}\right) \rightarrow C-1+\ln \left(\frac{1}{1-C}\right) \frac{1-C}{C} \equiv r_{0}(C)
$$

as $k_{m} \rightarrow \infty$, where $r_{0}(C) \rightarrow 0$ as $C \rightarrow 0$.

\section{References}

Abramowitz, M., and I. A. Stegun, eds. (1965). Handbook of Mathematical Functions with Formulas, Graphs, and Mathematical Tables. New York: Dover.

Awaya, Y., K. Iwasaki, and M. Watanabe. (2019). Rational Bubbles and Middlemen. Mimeo.

Awaya, Y., Z. Chen, and M. Watanabe. (2018). Intermediation and Reputation. Mimeo.

Biglaiser, G. (1993). "Middlemen as Experts," RAND Journal of Economics 24: 212-23.

Biglaiser, G., and F. Li. (2018). "Middlemen: The Good, the Bad, and the Ugly," RAND Journal of Economics 49 (1): 3-22.

Boehmer, E. (2005). “Dimensions of Execution Quality: Recent Evidence for US Equity Markets," Journal of Financial Economics 78: 553-82.

Caillaud, B., and B. Jullien. (2003). "Chicken and Egg: Competition among Intermediation Service Providers," RAND Journal of Economics 34: 521-52.

Condorelli, D., A. Galeotti, and V. Skreta. (2018). Selling through Referrals. London Business School Working Paper.

Dana, J. D., and K. E. Spier. (2001). "Revenue Sharing and Vertical Control in the Video Rental Industry," Journal of Industrial Economics 49 (3): 223-45.

Demsetz, H. (1968). “The Cost of Transacting," Quarterly Journal of Economics 82 (1): 33-53.

Duffie, D., N. Garleanu, and L. H. Pedersen. (2005). “Over-the-Counter Markets," Econometrica 73: 1815-47.

Fingleton, J. (1997a). "Competition Among Middlemen When Buyers And Sellers can Trade Directly," Journal of Industrial Economics XLV, 405-27, https://doi.org/10.1111/1467-6451. 00056.

Fingleton, J. (1997b). “Competition Between Intermediated And Direct Trade And the Timing of Disintermediation," Oxford Economic Papers 49: 543-56.

Galeotti, A., and J. L. Moraga-Gonzalez. (2009). "Platform Intermediation in a Market for Differentiated Products," European Economic Review 54: 417-28. 
Gautier, P., B. Hu, and M. Wantanabe. (2018). Marketmaking Middlemen. Tinbergen Institute Working Paper.

Geddes, K. O., M. L. Glasser, R. A. Moore, and T. C. Scott. (1990). "Evaluation of Classes of Definite Integrals Involving Elementary Functions via Differentiation of Special Functions," Applicable Algebra in Engineering, Communication and Computing 1: 149-65.

Gehrig, T. (1993). "Intermediation in Search Markets," Journal of Economics and Management Strategy 2 (1): 97-120.

Geromichalos, A., and K. M. Jung. (2018). "An Over-the-Counter Approach to the FOREX Market," International Economic Review 59 (2): 859-905.

Holzner, C., and M. Watanabe. (2018). Understanding the Role of the Public Employment Agency. Tinbergen Institute working paper.

Lagos, R., and S. Zhang. (2016). Monetary Exchange in Over-the-Counter Markets: A Theory of Speculative Bubbles, the FED Model, and Self-Fullfiling Liquidity Crises. NBER Working paper 21528.

Leslie, P. (2004). “Price Discrimination in Broadway Theater," RAND Journal of Economics 35 (3): 520-41.

Lester, B., G. Rocheteau, and P. Weill. (2014). “Competing for Order Flow in OTC Markets,” Journal of Money Credit and Banking 47: 77-126.

Li, F., C. Murry, C. Tian, and Y. Zhouy. (2019). The Price Theory and Empirics of Inventory Management. Mimeo.

Li, Y. (1998). “Middlemen and Private Information," Journal of Monetary Economics 42: 131-59.

Masters, A. (2007). “Middlemen in Search Equilibrium," International Economic Review 48: 343-62.

Moen, E. (1997). “Competitive Search Equilibrium,” Journal of Political Economy 105: 385-411.

Moraga-Gonzalez, J. L., and M. Wildenbeest. (2012). Comparison Sites, In Handbook of the Digital Economy, edited by P. Martin, and J. Waldfogel. Oxford University Press.

Mortensen, D., and R. Wright. (2002). “Competitive Pricing and Efficiency in Search Equilibrium," International Economic Review 43: 1-20.

Nosal, E., Y. Y. Wong, and R. Wright. (2015). “More on Middlemen: Equilibrium Entry and Effciency in Intermediated Markets," Journal of Money, Credit and Banking 47: 7-37.

Rhodes, A., M. Watanabe, and J. Zhou. (2019). Multiproduct intermediaries. Working paper, University of Yale.

Rubinstein, A., and A. Wolinsky. (1987). “Middlemen," Quarterly Journal of Economics 102: 581-93.

Rust, J., and R., Hall. (2003). “Middlemen versus Market Makers: A Theory of Competitive Exchange,” Journal of Political Economy 111: 353-403.

Sattinger, M. (2003). Brokers and the Equilibrium Price Function. SUNY Albany manuscript.

Shevichenko, A. (2004). “Middlemen," International Economic Review 45: 1-24.

Smith, E. (2004). "Intermediated Search," Economica 71: 619-36.

Spulber, D. F. (1996). “Market Making by Price Setting Firms," Review of Economic Studies 63: 559-80.

Spulber, D. F. (1999). Market Microstructure. Cambridge.

Stigler, G. J. (1964). "Public Regulation of the Securities Markets," Journal of Business, 117-42.

Temme, N. M. (1996). Special Functions: An Introduction to the Classical Functions of Mathematical Physics. New York: Wiley.

Watanabe, M. (2010). “A Model of Merchants,” Journal of Economic Theory, 145: 1865-89. 
Watanabe, M. (2018). “Middlemen: The Visible Market Makers,” Japanese Economic Review 69 (2): 156-70.

Wong, Y., and R. Wright. (2014). "Buyers, Sellers and Middlemen: Variations on Search-Theoretic Themes," International Economic Review 55 (2): 375-97. 